\title{
Local structure effects on heat transfer in very low tube-to-particle diameter ratio fixed beds of spheres
}

\author{
Anthony G. Dixon ${ }^{1 *}$
}

${ }^{1}$ Worcester Polytechnic Institute, Department of Chemical Engineering, 100 Institute Road, Worcester, Massachusetts 01609, USA.

${ }^{*}$ Corresponding author: agdixon@wpi.edu 
$99.8 \mathrm{~mm}$ column, $31.1 \mathrm{~mm}$ polystyrene spheres

\section{Format}

First 4 lines:

\# radial profiles \# radial T/C positions \# wall temperatures \# angular positions

Radial $\mathrm{T} / \mathrm{C}$ positions in $\mathrm{mm}$ from center of bed

Axial $\mathrm{T} / \mathrm{C}$ positions on tube wall (-ve means calming section) in $\mathrm{mm}$

Tube radius Sphere diameter

Data then follow in blocks:

$\operatorname{Re} \mathrm{L}(\mathrm{mm})$ Angle

$\mathrm{T}_{\text {in }}$ (entrance to calming section)

Tube wall temperature measurements

Radial profiles ( -1 means no temperature measured; note temperatures at $r=0.0$ were not used)

\begin{tabular}{|c|c|c|c|c|c|c|c|}
\hline 4 & 7 & 2 & & & & & \\
\hline 0.0 & 10.3 & 20.1 & 30.5 & 39.7 & 43.2 & 46.0 & \\
\hline-127.0 & -76.2 & -25.4 & -15.9 & -6.35 & 76.2 & 228.6 & 381.0 \\
\hline 49.9 & 31.7 & & & & & & \\
\hline $140 \quad 12$ & 50 & & & & & & \\
\hline 22.50 & & & & & & & \\
\hline 22.50 & 22.50 & 39.40 & 46.60 & 73.70 & 102.80 & 102.80 & 102.30 \\
\hline 41.20 & 37.40 & 30.20 & 40.20 & 64.30 & 64.10 & 73.70 & \\
\hline-1.00 & 38.10 & 45.80 & 49.80 & 55.90 & 77.10 & 78.80 & \\
\hline-1.00 & 45.40 & 48.70 & 50.10 & 56.00 & 62.30 & 76.20 & \\
\hline-1.00 & 42.10 & 39.30 & 45.40 & 47.70 & 67.50 & 79.70 & \\
\hline $\begin{array}{l}140 \quad 12 \\
22.50\end{array}$ & $5 \quad 45$ & & & & & & \\
\hline 22.50 & 22.90 & 40.60 & 47.60 & 74.20 & 102.80 & 102.80 & 102.60 \\
\hline 40.70 & 39.70 & 37.40 & 45.60 & 54.40 & 69.10 & 77.90 & \\
\hline-1.00 & 34.60 & 41.20 & 40.60 & 63.20 & 66.40 & 79.70 & \\
\hline-1.00 & 41.60 & 49.60 & 51.10 & 66.10 & 62.00 & 82.90 & \\
\hline-1.00 & 45.10 & 39.70 & 52.00 & 53.50 & 64.40 & 77.90 & \\
\hline $\begin{array}{l}18812 \\
23.10\end{array}$ & 545 & & & & & & \\
\hline 22.80 & 23.50 & 42.00 & 49.50 & 75.10 & 102.80 & 102.80 & 102.60 \\
\hline 39.20 & 38.20 & 37.00 & 46.10 & 63.40 & 66.70 & 84.40 & \\
\hline-1.00 & 34.60 & 47.40 & 39.10 & 72.50 & 66.10 & 83.50 & \\
\hline-1.00 & 39.90 & 46.40 & 51.30 & 61.00 & 63.80 & 74.80 & \\
\hline-1.00 & 42.00 & 38.20 & 52.20 & 55.40 & 73.40 & 81.90 & \\
\hline $\begin{array}{l}188 \quad 12 \\
23.10\end{array}$ & 50 & & & & & & \\
\hline 22.90 & 23.80 & 42.50 & 49.90 & 75.20 & 102.80 & 102.90 & 102.80 \\
\hline 41.30 & 35.40 & 36.10 & 41.00 & 52.00 & 80.20 & 76.60 & \\
\hline-1.00 & 43.20 & 48.80 & 63.50 & 60.70 & 70.30 & 77.90 & \\
\hline-1.00 & 45.50 & 47.40 & 47.20 & 58.20 & 61.10 & 74.30 & \\
\hline-1.00 & 39.70 & 40.40 & 45.90 & 52.20 & 61.80 & 77.50 & \\
\hline $\begin{array}{l}240 \quad 12 \\
23.30\end{array}$ & & & & & & & \\
\hline 22.90 & 24.10 & 43.00 & 50.40 & 75.50 & 102.60 & 102.80 & 102.60 \\
\hline 40.00 & 34.80 & 35.20 & 41.00 & 52.30 & 73.50 & 74.00 & \\
\hline
\end{tabular}




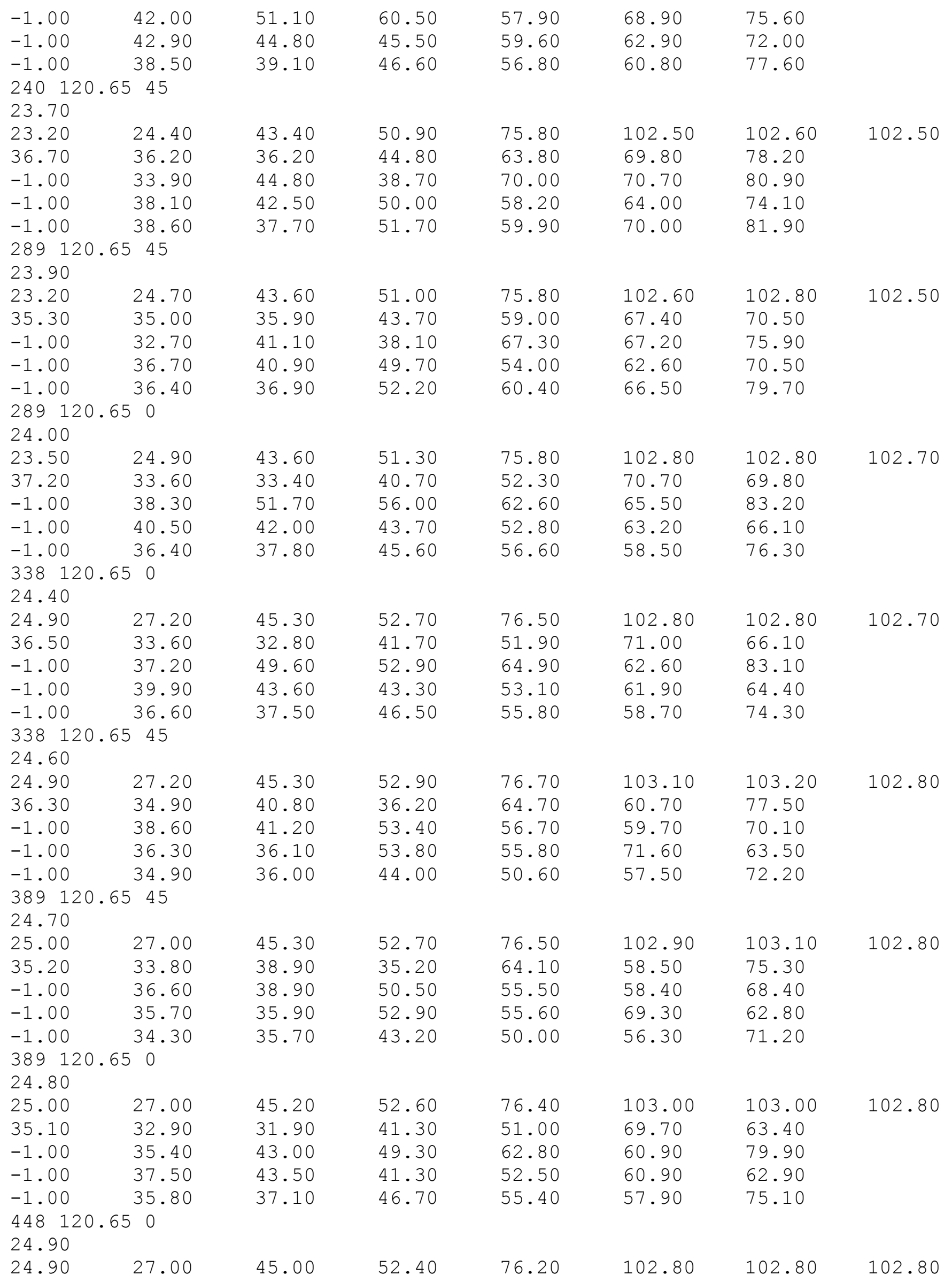




\begin{tabular}{|c|c|c|c|c|c|c|c|}
\hline 34.20 & 32.40 & 31.30 & 41.60 & 49.50 & 67.80 & 61.50 & \\
\hline-1.00 & 34.10 & 40.70 & 47.20 & 61.00 & 59.30 & 77.00 & \\
\hline-1.00 & 36.10 & 42.90 & 40.20 & 51.70 & 59.70 & 62.00 & \\
\hline-1.00 & 35.20 & 36.40 & 46.30 & 53.80 & 57.60 & 72.20 & \\
\hline \multicolumn{8}{|c|}{$\begin{array}{l}448120.65 \quad 45 \\
24.90\end{array}$} \\
\hline 24.90 & 27.00 & 44.80 & 52.40 & 76.20 & 102.80 & 102.80 & \multirow[t]{6}{*}{102.60} \\
\hline 34.20 & 33.20 & 36.90 & 36.10 & 60.20 & 58.90 & 68.40 & \\
\hline-1.00 & 35.50 & 38.00 & 46.70 & 56.50 & 57.50 & 68.60 & \\
\hline-1.00 & 35.00 & 35.20 & 53.40 & 54.30 & 69.50 & 61.30 & \\
\hline-1.00 & 33.80 & 35.20 & 43.90 & 50.80 & 53.80 & 70.20 & \\
\hline \multicolumn{7}{|c|}{$\begin{array}{l}494 \quad 120.65 \quad 45 \\
25.20\end{array}$} & \\
\hline 25.40 & 27.20 & 44.50 & 52.40 & 76.20 & 102.30 & 102.30 & \multirow[t]{5}{*}{102.30} \\
\hline 33.30 & 32.40 & 35.60 & 35.50 & 58.80 & 57.30 & 65.70 & \\
\hline-1.00 & 34.70 & 37.00 & 45.00 & 55.40 & 56.00 & 66.90 & \\
\hline-1.00 & 34.40 & 35.10 & 52.60 & 53.70 & 68.20 & 60.50 & \\
\hline-1.00 & 33.40 & 34.90 & 43.10 & 50.60 & 52.90 & 69.00 & \\
\hline \multicolumn{8}{|c|}{$\begin{array}{l}494120.650 \\
24.90\end{array}$} \\
\hline 25.20 & 27.20 & 44.50 & 52.40 & 76.10 & 102.60 & 102.60 & \multirow[t]{6}{*}{102.60} \\
\hline 33.30 & 32.10 & 31.10 & 41.40 & 49.00 & 67.20 & 60.20 & \\
\hline-1.00 & 33.50 & 38.60 & 44.90 & 57.90 & 58.50 & 73.50 & \\
\hline-1.00 & 35.30 & 43.30 & 39.20 & 51.50 & 58.40 & 60.50 & \\
\hline-1.00 & 34.90 & 35.80 & 45.90 & 53.50 & 56.60 & 71.90 & \\
\hline \multicolumn{7}{|c|}{$\begin{array}{l}549120.650 \\
25.30\end{array}$} & \\
\hline 25.30 & 27.30 & 44.20 & 52.50 & 75.90 & 102.50 & 102.60 & \multirow[t]{5}{*}{102.50} \\
\hline 32.80 & 31.60 & 30.90 & 41.40 & 48.40 & 65.20 & 58.40 & \\
\hline-1.00 & 32.90 & 37.50 & 43.90 & 56.50 & 57.10 & 71.40 & \\
\hline-1.00 & 34.70 & 42.40 & 38.70 & 50.80 & 58.00 & 59.40 & \\
\hline-1.00 & 34.40 & 35.50 & 45.20 & 52.70 & 56.00 & 70.10 & \\
\hline \multicolumn{8}{|c|}{$\begin{array}{l}549120.65 \quad 45 \\
25.30\end{array}$} \\
\hline 25.30 & 27.30 & 44.20 & 52.50 & 76.10 & 102.80 & 102.80 & \multirow[t]{5}{*}{102.80} \\
\hline 32.20 & 33.70 & 35.10 & 41.00 & 49.70 & 59.40 & 62.60 & \\
\hline-1.00 & 31.00 & 33.50 & 44.20 & 59.50 & 61.90 & 64.40 & \\
\hline-1.00 & 33.00 & 34.90 & 43.30 & 49.90 & 56.00 & 62.60 & \\
\hline-1.00 & 34.70 & 36.20 & 47.50 & 54.60 & 63.20 & 67.30 & \\
\hline \multirow{2}{*}{\multicolumn{8}{|c|}{$\begin{array}{l}607 \quad 120.6545 \\
24.80\end{array}$}} \\
\hline 24.80 & & & & & & & \\
\hline 25.00 & 27.20 & 44.00 & 52.20 & 75.90 & 102.50 & 102.50 & \multirow[t]{5}{*}{102.50} \\
\hline 31.80 & 33.00 & 34.80 & 39.60 & 47.90 & 53.70 & 60.00 & \\
\hline-1.00 & 30.50 & 39.10 & 37.50 & 61.50 & 54.50 & 65.20 & \\
\hline-1.00 & 33.80 & 34.80 & 45.80 & 48.50 & 54.00 & 60.90 & \\
\hline-1.00 & 34.30 & 35.90 & 47.90 & 54.00 & 61.00 & 65.70 & \\
\hline \multirow{2}{*}{\multicolumn{8}{|c|}{$\begin{array}{l}607 \quad 120.650 \\
25.40\end{array}$}} \\
\hline & & & & & & & \\
\hline 25.40 & 27.30 & 44.10 & 52.50 & 76.10 & 102.70 & 102.70 & \multirow[t]{6}{*}{102.70} \\
\hline 32.40 & 31.20 & 30.20 & 40.60 & 48.50 & 63.50 & 58.50 & \\
\hline-1.00 & 32.10 & 37.10 & 44.10 & 57.20 & 56.20 & 72.00 & \\
\hline-1.00 & 34.00 & 40.10 & 39.10 & 48.20 & 57.50 & 57.30 & \\
\hline-1.00 & 34.00 & 35.00 & 43.40 & 49.50 & 55.20 & 64.40 & \\
\hline 14016 & 0 & & & & & & \\
\hline
\end{tabular}

23.30 


\begin{tabular}{|c|c|c|c|c|c|c|c|}
\hline 24.00 & 26.30 & 47.10 & 53.30 & 77.60 & 103.60 & 103.70 & 103.50 \\
\hline 54.90 & 54.60 & 62.30 & 59.20 & 82.30 & 82.20 & 93.40 & \\
\hline-1.00 & 63.40 & 63.40 & 62.40 & 72.40 & 79.10 & 89.50 & \\
\hline-1.00 & 54.10 & 45.80 & 64.30 & 59.20 & 61.60 & 65.20 & \\
\hline-1.00 & 46.30 & 53.70 & 64.10 & 86.30 & 71.40 & 93.70 & \\
\hline $\begin{array}{l}140165.1 \\
23.50\end{array}$ & 45 & & & & & & \\
\hline 24.00 & 26.50 & 47.30 & 53.30 & 77.60 & 103.40 & 103.40 & 103.40 \\
\hline 54.90 & 50.40 & 50.80 & 61.70 & 68.40 & 83.80 & 81.30 & \\
\hline-1.00 & 61.00 & 65.40 & 62.40 & 69.20 & 71.70 & 74.90 & \\
\hline-1.00 & 60.30 & 62.20 & 65.40 & 69.00 & 57.60 & 83.10 & \\
\hline-1.00 & 52.20 & 61.70 & 51.60 & 62.80 & 84.90 & 82.80 & \\
\hline $\begin{array}{l}188165.1 \\
23.50\end{array}$ & 45 & & & & & & \\
\hline 23.90 & 26.50 & 47.10 & 53.20 & 77.30 & 102.10 & 102.30 & 102.30 \\
\hline 51.00 & 48.50 & 49.00 & 65.10 & 67.60 & 80.50 & 81.90 & \\
\hline-1.00 & 53.40 & 63.70 & 58.50 & 69.30 & 71.30 & 72.50 & \\
\hline-1.00 & 57.80 & 53.10 & 62.30 & 68.30 & 58.00 & 79.60 & \\
\hline-1.00 & 49.90 & 66.10 & 51.30 & 60.00 & 84.00 & 79.40 & \\
\hline $188 \quad 165.1$ & 0 & & & & & & \\
\hline 23.90 & & & & & & & \\
\hline 24.30 & 26.70 & 47.00 & 53.20 & 77.30 & 102.40 & 102.50 & 102.30 \\
\hline 50.20 & 49.00 & 58.60 & 52.90 & 76.60 & 73.90 & 87.20 & \\
\hline-1.00 & 59.10 & 63.50 & 62.30 & 71.30 & 70.50 & 75.00 & \\
\hline-1.00 & 51.70 & 42.60 & 60.60 & 55.90 & 58.50 & 58.50 & \\
\hline-1.00 & 45.50 & 50.20 & 61.20 & 79.30 & 69.10 & 86.70 & \\
\hline $240 \quad 165.1$ & 0 & & & & & & \\
\hline 23.90 & & & & & & & \\
\hline 24.40 & 26.70 & 47.00 & 53.20 & 77.00 & 102.30 & 102.50 & 102.10 \\
\hline 47.90 & 45.70 & 53.60 & 51.80 & 73.60 & 71.70 & 83.50 & \\
\hline-1.00 & 54.40 & 61.00 & 60.50 & 69.20 & 76.10 & 72.80 & \\
\hline-1.00 & 48.00 & 42.00 & 62.20 & 57.80 & 65.80 & 58.90 & \\
\hline-1.00 & 43.80 & 48.00 & 62.20 & 75.20 & 64.40 & 83.40 & \\
\hline $\begin{array}{l}240165.1 \\
23.90\end{array}$ & 45 & & & & & & \\
\hline 24.30 & 26.70 & 46.70 & 53.10 & 76.80 & 102.00 & 102.30 & 102.00 \\
\hline 46.80 & 45.20 & 46.20 & 62.00 & 61.30 & 77.90 & 75.20 & \\
\hline-1.00 & 48.00 & 58.90 & 55.40 & 66.70 & 69.60 & 69.70 & \\
\hline-1.00 & 53.60 & 48.00 & 54.50 & 64.90 & 58.90 & 77.90 & \\
\hline-1.00 & 43.80 & 56.50 & 59.90 & 67.60 & 79.40 & 79.30 & \\
\hline $\begin{array}{l}289165.1 \\
24.30\end{array}$ & 45 & & & & & & \\
\hline 24.60 & 26.90 & 46.70 & 53.10 & 76.80 & 102.10 & 102.10 & 102.10 \\
\hline 44.80 & 43.30 & 43.90 & 60.40 & 59.20 & 76.50 & 72.30 & \\
\hline-1.00 & 45.30 & 56.00 & 54.30 & 65.40 & 66.70 & 68.10 & \\
\hline-1.00 & 51.90 & 48.70 & 50.80 & 67.20 & 59.20 & 81.40 & \\
\hline-1.00 & 42.10 & 53.20 & 61.10 & 69.70 & 77.60 & 79.70 & \\
\hline 289165.1 & 0 & & & & & & \\
\hline 24.60 & & & & & & & \\
\hline 24.60 & 27.20 & 46.70 & 53.20 & 76.90 & 102.20 & 102.20 & 102.20 \\
\hline 44.70 & 42.80 & 51.40 & 48.70 & 70.60 & 70.50 & 81.70 & \\
\hline-1.00 & 51.40 & 59.20 & 55.40 & 67.40 & 77.90 & 72.90 & \\
\hline-1.00 & 45.80 & 42.60 & 62.90 & 58.30 & 65.80 & 58.10 & \\
\hline-1.00 & 42.10 & 45.30 & 57.90 & 72.10 & 60.80 & 80.00 & \\
\hline $\begin{array}{lll}338 & 165.1\end{array}$ & 0 & & & & & & \\
\hline
\end{tabular}


24.60

24.80

43.50

$-1.00$

27.40

41.70

$-1.00$

50.70

$-1.00$

44.80

3381

24.80

25.10

42.80

$-1.00$

$-1.00$

$-1.00$

389165.145

24.80

25.10

41.20

$-1.00$

$-1.00$

$-1.00$

389165.10

24.90

25.20

41.50

$-1.00$

$-1.00$

$-1.00$

41.30

$448 \quad 165.10$

25.10

25.40

40.80

$-1.00$

$-1.00$

$-1.00$

$\begin{array}{lll}448 & 165.1 \quad 45\end{array}$

25.10

25.20

39.90

27.50

$-1.00$

39.10

$-1.00$

40.90

$-1.00$

43.10

$494 \quad 165.145$

25.20

25.40

39.30

27.50

$-1.00$

38.70

$-1.00$

40.20

$-1.00$

41.50

$494 \quad 165.10$

25.20

25.40

39.60

$-1.00$

$-1.00$

$-1.00$

\begin{abstract}
46.30
51.90

57.60

42.60

44.90
\end{abstract}

46.30

42.10

52.50

47.40

52.20

45.80

41.20

49.50

47.20

51.10

45.50

52.20

55.10

42.00

43.60

45.30

51.50

51.40

41.90

43.10

45.00

40.10

48.30

49.90

47.50

45.00

39.60

46.50

49.50

47.00

44.90

51.10

47.70

42.10

42.30
53.20

47.60

54.00

61.80

57.60

53.20

57.80

54.00

46.70

58.70

53.20

56.30

54.10

43.90

56.00

52.90

45.50

50.10

59.10

53.50

53.10
45.40
48.50
57.60
52.80

52.90

57.00

54.00

50.60

57.90

52.90

56.30

53.80

48.20

55.60

52.90

44.50

46.90

55.70

50.80
76.90

69.70

66.10

57.70

70.50

76.60

58.10

62.60

65.40

67.90

76.60

57.50

61.00

63.40

67.30

76.30

69.40

66.60

57.20

68.90

76.30

69.30

64.70

55.70

67.30

76.10

52.20

64.10

65.00

61.00

76.10

52.00

62.80

64.10

60.50

76.10

67.70

63.90

55.70

65.80
102.20

69.30

74.10

66.70

59.40

102.20

74.70

64.20

58.60

75.50

102.20

72.90

62.30

57.80

73.30

102.10

71.10

69.60

60.50

57.10

102.00

70.50

68.00

59.90

57.20

102.10

74.40

67.10

59.00

70.70

101.90

73.50

66.90

58.40

70.10

102.20

70.80

66.70

56.20

56.40
102.20

79.10

71.20

58.90

78.20

102.20

69.40

65.80

78.70

77.50

102.20

68.60

64.60

77.30

76.40

102.20

79.90

71.00

58.70

75.10

102.00

78.20

69.20

58.40

73.90

102.10

62.40

70.80

73.10

66.00

101.90

61.00

70.00

71.90

66.50 
549165.10

25.40

25.60

39.30

27.60

44.80

52.80

75.80

101.90

102.00

101.90

$-1.00$

50.20

44.40

67.60

69.40

78.20

46.70

62.70

41.30

$-1.00$

39.80

$-1.00$

38.60

54.70

54.80

65.70

69.70

42.30

50.80

65.20

56.40

58.10

55.70

71.90

549.1
25.40

25.40

27.60

44.60

52.50

75.80

101.90

101.90

101.90

39.00

52.50

71.40

62.00

53.20

49.30

$-1.00$

39.50

44.50

$-1.00$

41.30

46.90

61.00

64.70

66.80

65.80

55.70

75.50

51.90

61.70

68.40

68.60

$607 \quad 165.145$

24.30

22.60

22.50

34.00

42.10

70.00

102.40

102.40

102.40

35.20

49.60

68.10

57.20

50.10

57.00

62.50

65.30

46.70

61.50

52.80

70.70

42.30

59.00

66.00

67.50

607165.10

24.40

23.00

23.00

35.60

44.00

71.30

102.80

102.80

102.50

41.70

65.40

64.90

71.60

41.50

59.00

62.40

65.70

52.00

49.60

59.00

53.70

39.60

50.30

$140 \quad 215.90$

23.40

23.70

25.70

46.10

61.70

52.60

77.20

63.30

69.00

70.50

63.50

$62.10 \quad 64.90$

68.10

61.70

79.10

53.60

70.70

$-1.00$

56.60

$140 \quad 215.9 \quad 45$

23.70

23.70

26.00

46.10

52.60

77.30

71.00

69.40

74.10

71.90

59.80

$77 \cdot 00$

103.60

76.90

76.00

87.90

83.00

61.70

52.80

62.70

68.10

$188 \quad 215.9 \quad 45$

23.70

23.80

26.00

46.30

52.60

77.30

103.60

103.60

103.40

59.10

66.40

74.10

70.50

78.10

65.40

73.70

90.20

86.70

51.70

71.90

78.90

84.40

67.50

75.30

76.80

87.30

$\begin{array}{lll}188 & 215.9 & 0\end{array}$

23.50

24.00

26.20

46.40

52.70

77.20

103.50

103.70

103.40

55.70

58.20

61.80

66.10

66.20

77.00

68.00

64.30

81.60

75.30

89.40

62.70

63.80

79.70

82.90

84.80 


\begin{tabular}{|c|c|c|c|c|c|c|c|}
\hline $\begin{array}{l}-1.00 \\
240215.9 \\
24.10\end{array}$ & $\begin{array}{l}60.10 \\
0\end{array}$ & 56.20 & 64.70 & 76.00 & 74.10 & 87.10 & \\
\hline 25.00 & 27.30 & 46.50 & 53.40 & 77.50 & 103.50 & 103.90 & 103.40 \\
\hline 50.80 & 53.80 & 56.60 & 68.30 & 61.20 & 70.50 & 66.10 & \\
\hline-1.00 & 51.10 & 45.50 & 57.50 & 78.50 & 71.30 & 84.40 & \\
\hline-1.00 & 49.00 & 58.50 & 59.10 & 73.40 & 77.10 & 80.20 & \\
\hline-1.00 & 54.30 & 51.10 & 64.10 & 70.10 & 74.00 & 82.60 & \\
\hline $\begin{array}{l}240215.9 \\
24.10\end{array}$ & 45 & & & & & & \\
\hline 25.00 & 27.30 & 46.50 & 53.40 & 77.50 & 103.70 & 103.70 & 103.50 \\
\hline 51.00 & 53.50 & 53.10 & 55.40 & 72.00 & 64.40 & 76.80 & \\
\hline-1.00 & 52.50 & 55.40 & 61.60 & 67.20 & 89.00 & 79.00 & \\
\hline-1.00 & 49.30 & 47.20 & 58.90 & 67.80 & 76.60 & 86.70 & \\
\hline-1.00 & 54.90 & 58.00 & 65.00 & 76.60 & 67.90 & 89.90 & \\
\hline $\begin{array}{l}289215.9 \\
24.40\end{array}$ & 45 & & & & & & \\
\hline 25.00 & 27.60 & 46.50 & 53.40 & 77.50 & 103.70 & 103.70 & 103.70 \\
\hline 50.20 & 51.70 & 52.20 & 54.50 & 71.70 & 65.70 & 75.40 & \\
\hline-1.00 & 52.00 & 55.70 & 61.80 & 67.60 & 88.10 & 80.90 & \\
\hline-1.00 & 48.50 & 46.60 & 59.80 & 66.50 & 77.50 & 86.70 & \\
\hline-1.00 & 53.60 & 55.40 & 64.90 & 75.00 & 69.60 & 87.60 & \\
\hline $\begin{array}{l}289215.9 \\
24.40\end{array}$ & 0 & & & & & & \\
\hline 25.00 & 27.60 & 46.50 & 53.40 & 77.50 & 103.70 & 103.70 & 103.70 \\
\hline 49.80 & 52.50 & 55.20 & 66.90 & 60.60 & 67.80 & 67.20 & \\
\hline-1.00 & 50.00 & 44.80 & 56.60 & 78.20 & 70.40 & 81.90 & \\
\hline-1.00 & 47.50 & 56.90 & 58.60 & 71.60 & 77.10 & 80.30 & \\
\hline-1.00 & 52.90 & 50.00 & 63.70 & 69.50 & 73.20 & 83.10 & \\
\hline $\begin{array}{l}338215.9 \\
24.50\end{array}$ & 0 & & & & & & \\
\hline 25.10 & 27.60 & 46.50 & 53.40 & 77.50 & 103.70 & 103.70 & 103.50 \\
\hline 48.70 & 51.30 & 54.50 & 66.00 & 60.80 & 67.10 & 65.80 & \\
\hline-1.00 & 48.80 & 44.10 & 55.90 & 76.20 & 68.80 & 79.60 & \\
\hline-1.00 & 46.20 & 56.40 & 56.80 & 72.20 & 76.60 & 80.80 & \\
\hline-1.00 & 50.80 & 49.00 & 62.90 & 68.90 & 72.90 & 81.70 & \\
\hline $\begin{array}{l}338215.9 \\
24.80\end{array}$ & 45 & & & & & & \\
\hline 25.30 & 27.60 & 46.30 & 53.40 & 77.40 & 103.40 & 103.50 & 103.40 \\
\hline 47.90 & 49.00 & 50.20 & 53.80 & 70.40 & 64.70 & 74.20 & \\
\hline-1.00 & 50.20 & 52.60 & 61.00 & 65.80 & 85.30 & 79.30 & \\
\hline-1.00 & 46.40 & 44.80 & 57.30 & 63.20 & 71.60 & 82.50 & \\
\hline-1.00 & 50.10 & 52.50 & 62.80 & 72.90 & 71.20 & 85.20 & \\
\hline $\begin{array}{l}389215.9 \\
24.80\end{array}$ & 45 & & & & & & \\
\hline 25.00 & 27.60 & 46.10 & 53.40 & 77.30 & 103.20 & 103.40 & 103.20 \\
\hline 46.30 & 47.10 & 48.50 & 53.20 & 68.80 & 63.40 & 72.50 & \\
\hline-1.00 & 49.00 & 51.30 & 59.60 & 64.50 & 82.60 & 78.40 & \\
\hline-1.00 & 45.00 & 43.80 & 57.30 & 61.40 & 71.60 & 81.20 & \\
\hline-1.00 & 47.90 & 50.70 & 61.20 & 70.60 & 70.10 & 83.10 & \\
\hline $\begin{array}{l}389215.9 \\
24.80\end{array}$ & 0 & & & & & & \\
\hline 25.10 & 27.40 & 45.70 & 53.30 & 77.00 & 103.20 & 103.20 & 103.20 \\
\hline 46.10 & 48.00 & 53.50 & 60.70 & 62.20 & 69.30 & 64.90 & \\
\hline-1.00 & 47.00 & 44.00 & 55.60 & 79.20 & 67.60 & 86.30 & \\
\hline
\end{tabular}




\begin{tabular}{|c|c|c|c|c|c|c|c|}
\hline-1.00 & 43.80 & 58.00 & 51.30 & 67.80 & 74.80 & 73.80 & \\
\hline-1.00 & 48.50 & 46.60 & 59.10 & 62.00 & 70.70 & 75.90 & \\
\hline $\begin{array}{l}448215.9 \\
24.80\end{array}$ & 0 & & & & & & \\
\hline 25.20 & 27.40 & 45.60 & 53.10 & 76.80 & 102.90 & 103.00 & 102.90 \\
\hline 44.70 & 46.40 & 52.60 & 58.40 & 61.10 & 68.10 & 63.80 & \\
\hline-1.00 & 45.70 & 42.90 & 54.90 & 77.10 & 66.20 & 84.00 & \\
\hline-1.00 & 42.40 & 56.30 & 49.90 & 67.30 & 72.50 & 72.80 & \\
\hline-1.00 & 46.60 & 45.70 & 56.80 & 61.40 & 69.10 & 74.70 & \\
\hline $\begin{array}{l}448215.9 \\
25.00\end{array}$ & 45 & & & & & & \\
\hline 25.30 & 27.60 & 45.10 & 53.10 & 76.80 & 103.10 & 103.20 & 102.90 \\
\hline 44.20 & 44.80 & 46.60 & 53.80 & 66.70 & 61.10 & 69.90 & \\
\hline-1.00 & 46.60 & 48.50 & 57.60 & 60.90 & 75.10 & 74.10 & \\
\hline-1.00 & 42.70 & 42.60 & 52.20 & 61.90 & 74.00 & 81.70 & \\
\hline-1.00 & 45.40 & 48.00 & 57.70 & 68.80 & 69.80 & 80.60 & \\
\hline $\begin{array}{l}494215.9 \\
25.00\end{array}$ & 45 & & & & & & \\
\hline 25.30 & 27.30 & 45.10 & 53.00 & 76.70 & 102.90 & 102.90 & 102.90 \\
\hline 43.40 & 44.10 & 46.10 & 53.60 & 66.10 & 60.50 & 69.40 & \\
\hline-1.00 & 46.20 & 47.90 & 57.20 & 60.10 & 73.70 & 73.00 & \\
\hline-1.00 & 42.10 & 42.30 & 51.70 & 60.80 & 73.10 & 81.40 & \\
\hline-1.00 & 44.60 & 47.40 & 57.50 & 68.10 & 69.60 & 79.10 & \\
\hline $\begin{array}{l}494215.9 \\
25.00\end{array}$ & 0 & & & & & & \\
\hline 25.50 & 27.60 & 45.10 & 53.00 & 76.80 & 102.90 & 102.90 & 102.90 \\
\hline 43.70 & 46.20 & 49.80 & 61.00 & 56.60 & 62.80 & 61.50 & \\
\hline-1.00 & 43.70 & 40.10 & 53.10 & 66.30 & 65.50 & 72.00 & \\
\hline-1.00 & 41.80 & 50.40 & 51.70 & 67.80 & 71.70 & 79.10 & \\
\hline-1.00 & 45.20 & 44.80 & 59.60 & 66.10 & 68.50 & 77.90 & \\
\hline 549215.9 & 0 & & & & & & \\
\hline 25.00 & & & & & & & \\
\hline 25.50 & 27.50 & 44.70 & 53.00 & 76.30 & 102.90 & 102.90 & 102.90 \\
\hline 42.80 & 45.20 & 49.10 & 59.60 & 56.20 & 62.50 & 61.20 & \\
\hline-1.00 & 42.90 & 39.60 & 52.60 & 64.70 & 65.00 & 70.40 & \\
\hline-1.00 & 41.00 & 49.60 & 50.80 & 66.70 & 70.50 & 76.90 & \\
\hline-1.00 & 44.30 & 44.20 & 59.10 & 65.70 & 67.30 & 77.50 & \\
\hline $\begin{array}{l}549215.9 \\
25.30\end{array}$ & 45 & & & & & & \\
\hline 25.50 & 27.50 & 44.70 & 53.00 & 76.30 & 103.00 & 103.20 & 102.90 \\
\hline 42.30 & 43.90 & 47.80 & 56.30 & 58.50 & 70.70 & 67.50 & \\
\hline-1.00 & 41.60 & 42.10 & 54.20 & 61.60 & 72.20 & 84.40 & \\
\hline-1.00 & 42.90 & 45.20 & 54.00 & 67.20 & 68.80 & 76.30 & \\
\hline-1.00 & 43.00 & 44.70 & 55.80 & 64.60 & 58.70 & 69.70 & \\
\hline $\begin{array}{l}607215.9 \\
24.80\end{array}$ & 45 & & & & & & \\
\hline 25.50 & 27.60 & 44.70 & 52.80 & 76.30 & 103.20 & 103.20 & 102.90 \\
\hline 41.80 & 43.50 & 47.50 & 55.50 & 58.00 & 69.40 & 67.00 & \\
\hline-1.00 & 40.70 & 41.40 & 54.10 & 58.80 & 71.60 & 82.40 & \\
\hline-1.00 & 42.40 & 44.40 & 53.70 & 66.00 & 67.80 & 75.20 & \\
\hline-1.00 & 42.10 & 43.80 & 54.90 & 63.50 & 58.40 & 68.70 & \\
\hline $\begin{array}{l}607215.9 \\
25.30\end{array}$ & 0 & & & & & & \\
\hline 25.50 & 27.60 & 44.40 & 52.70 & 76.30 & 103.20 & 103.20 & 102.90 \\
\hline 41.80 & 42.90 & 48.80 & 54.30 & 58.80 & 66.40 & 61.50 & \\
\hline
\end{tabular}


$-1.00$

$-1.00$

$-1.00$
42.30

39.90

43.40
40.40

52.60

42.90
52.60

46.20

52.70
72.40

66.10

58.60
62.70

67.80

66.00

78.90

71.70

71.50 


\section{$75.4 \mathrm{~mm}$ column, $25.5 \mathrm{~mm}$ polystyrene spheres}

\section{Format}

First 4 lines:

\# radial profiles \# radial T/C positions \# wall temperatures \# angular positions

Radial $\mathrm{T} / \mathrm{C}$ positions in $\mathrm{mm}$ from center of bed

Axial $\mathrm{T} / \mathrm{C}$ positions on tube wall (-ve means calming section) in $\mathrm{mm}$

Tube radius Sphere diameter

Data then follow in blocks:

$\operatorname{Re} \quad L(\mathrm{~mm})$ Angle

$\mathrm{T}_{\text {in }}$ (entrance to calming section)

Tube wall temperature measurements

Radial profiles (-1 means no temperature measured; note temperatures at $r=0.0$ were not used)

\begin{tabular}{|c|c|c|c|c|c|c|c|}
\hline \multicolumn{8}{|l|}{4} \\
\hline \multicolumn{8}{|c|}{10.3} \\
\hline \multirow{2}{*}{\multicolumn{8}{|c|}{$\begin{array}{l}-127.0 \\
37.5\end{array}$}} \\
\hline & & & & & & & \\
\hline \multicolumn{8}{|c|}{20898.4250} \\
\hline \multicolumn{8}{|c|}{23.40} \\
\hline 23.60 & 23.40 & 33.00 & 44.40 & 62.20 & 102.50 & 103.00 & \multirow[t]{5}{*}{102.70} \\
\hline 65.90 & 54.40 & 52.80 & 64.10 & 68.40 & 70.30 & 88.10 & \\
\hline-1.00 & 56.00 & 61.80 & 60.80 & 72.30 & 77.20 & 81.00 & \\
\hline-1.00 & 61.10 & 63.70 & 63.80 & 71.40 & 74.90 & 83.00 & \\
\hline-1.00 & 61.50 & 60.90 & 66.30 & 68.70 & 79.90 & 82.30 & \\
\hline \multicolumn{8}{|c|}{$20898.425 \quad 45$} \\
\hline 23.50 & 23.50 & 33.80 & 45.00 & 62.50 & 102.60 & 102.80 & \multirow[t]{5}{*}{102.60} \\
\hline 67.30 & 55.10 & 58.70 & 62.70 & 66.60 & 77.60 & 85.70 & \\
\hline-1.00 & 61.30 & 62.70 & 65.70 & 70.90 & 75.00 & 81.70 & \\
\hline-1.00 & 62.00 & 64.20 & 69.50 & 72.30 & 78.80 & 90.60 & \\
\hline-1.00 & 60.70 & 55.10 & 66.10 & 76.90 & 77.30 & 87.50 & \\
\hline \multicolumn{8}{|c|}{$25298.425 \quad 45$} \\
\hline \multicolumn{8}{|c|}{23.80} \\
\hline 23.80 & 23.80 & 33.60 & 44.40 & 61.90 & 102.60 & 103.10 & \multirow[t]{5}{*}{102.80} \\
\hline 66.60 & 54.40 & 57.30 & 61.10 & 65.00 & 76.90 & 84.00 & \\
\hline-1.00 & 59.90 & 61.20 & 64.80 & 68.90 & 73.30 & 79.00 & \\
\hline-1.00 & 60.70 & 62.50 & 67.50 & 70.80 & 76.20 & 89.50 & \\
\hline-1.00 & 59.60 & 55.20 & 65.00 & 74.30 & 74.70 & 85.40 & \\
\hline \multicolumn{8}{|c|}{25298.4250} \\
\hline 24.00 & 24.00 & 33.50 & 44.50 & 62.00 & 102.80 & 102.90 & \multirow[t]{5}{*}{102.90} \\
\hline 66.70 & 54.70 & 54.00 & 64.30 & 67.50 & 68.00 & 85.40 & \\
\hline-1.00 & 56.50 & 61.40 & 60.40 & 72.50 & 74.60 & 80.90 & \\
\hline-1.00 & 61.00 & 63.40 & 62.90 & 69.90 & 73.30 & 80.00 & \\
\hline-1.00 & 61.60 & 60.70 & 65.20 & 69.90 & 77.70 & 81.90 & \\
\hline \multirow{2}{*}{\multicolumn{8}{|c|}{$\begin{array}{l}29698.4250 \\
24.00\end{array}$}} \\
\hline & & & & & & & \\
\hline 24.20 & 24.00 & 32.80 & 43.10 & 60.60 & 102.80 & 103.00 & 102.80 \\
\hline
\end{tabular}




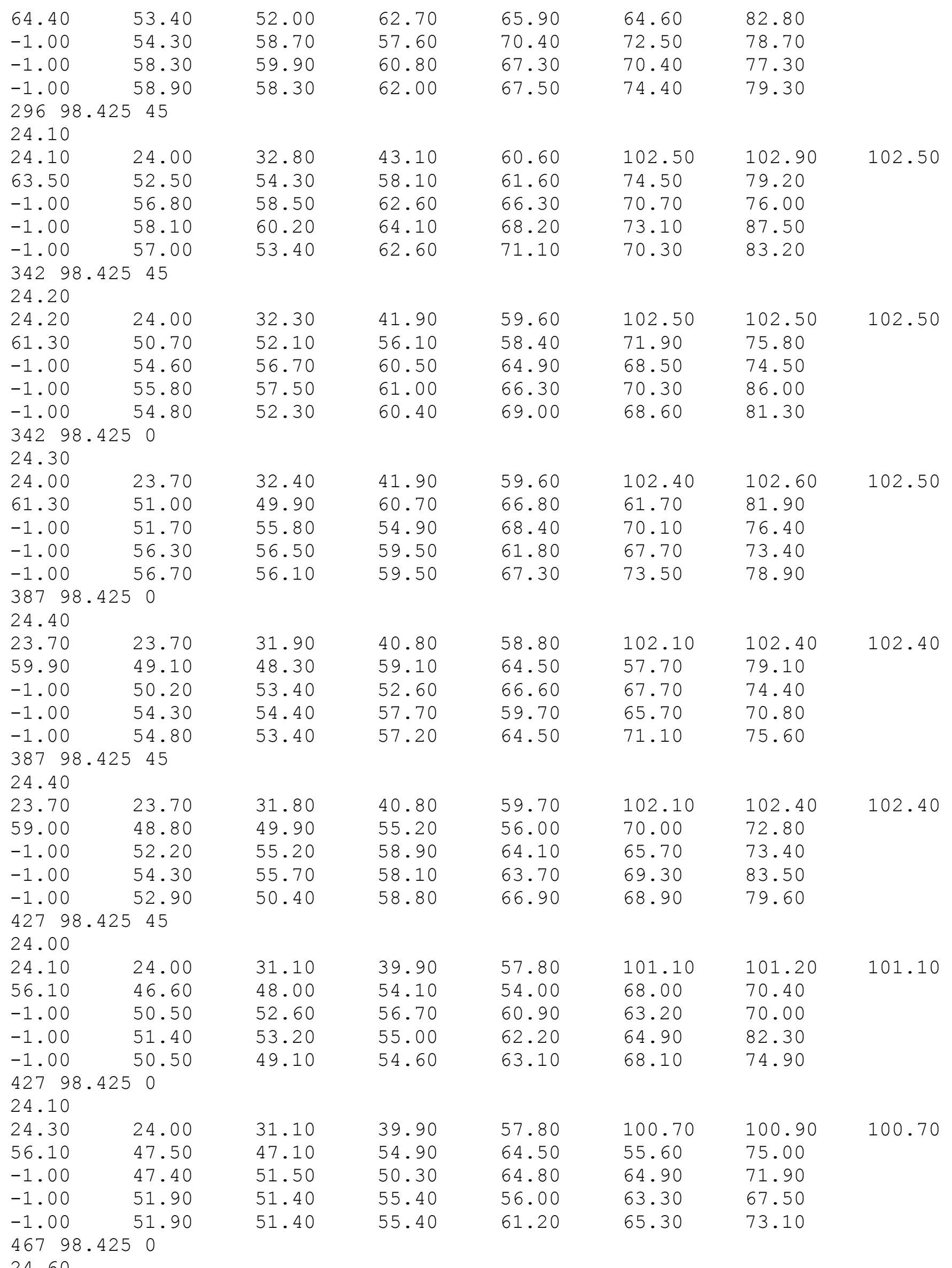




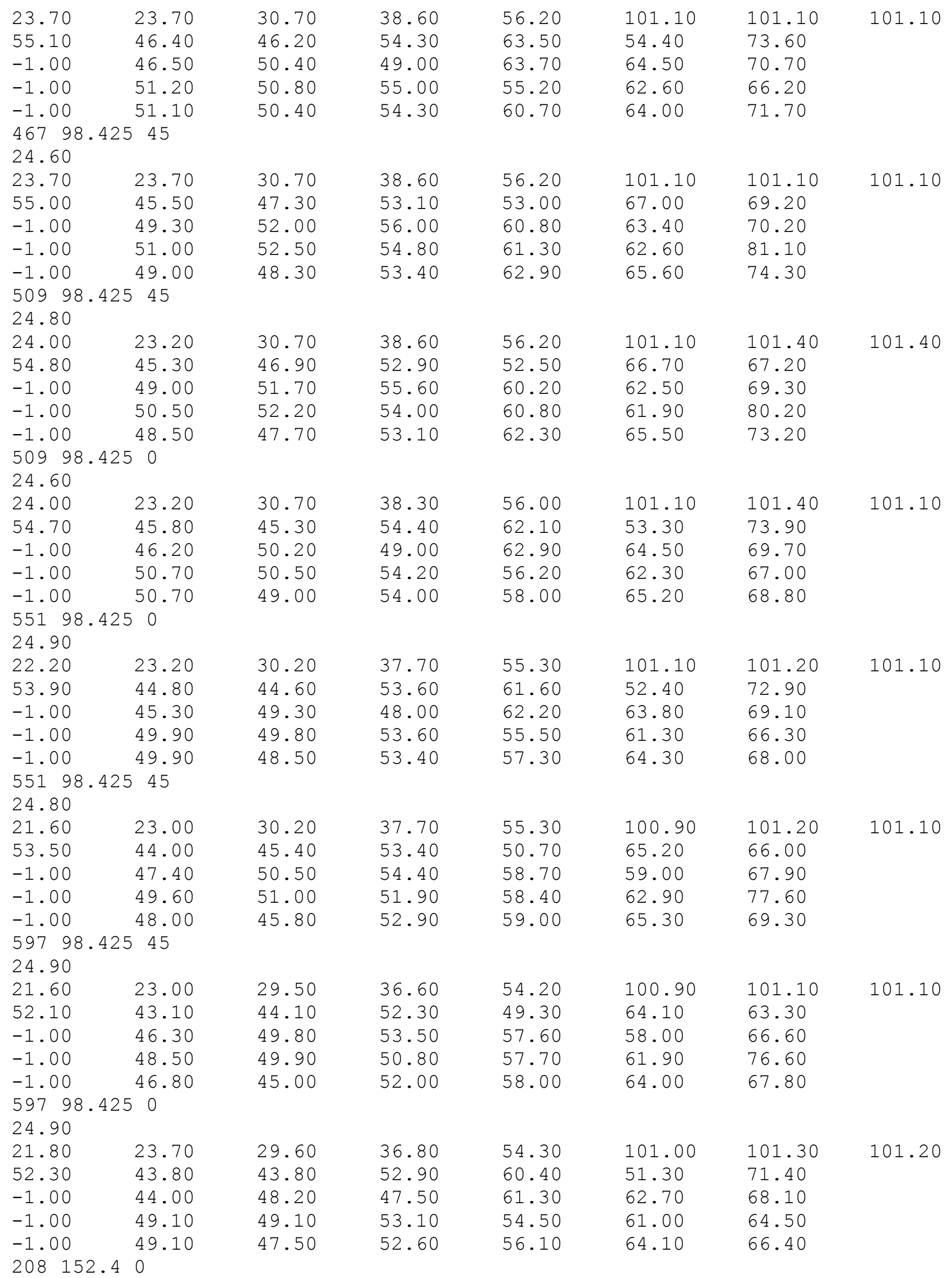


21.80

22.10

79.40

22.10

33.00

79.70

76.60

$-1.00$

75.20

77.90

$-1.00$

75.60

72.10

44.20
80.00
80.00
78.10
79.30

62.00

85.30

101.60

101.80

101.80

$-1.00$

73.40

$\begin{array}{lll}208 & 152.4 \quad 45\end{array}$

22.10

22.30

22.10

32.90

44.00

80.00

79.30

$-1.00$

77.40

77.70

72.70

76.10

80.00

83.50

86.30

92.60

83.70

85.50

89.10

82.00

90.70

95.70

87.80

89.30

$-1.00$

75.50

76.10

74.40

62.00

101.80

101.80

101.80

81.90

87.20

91.80

84.00

88.10

89.00

86.90

80.00

96.40

$-1.00$

70.10

83.10

85.30

93.10

21.90

22.30

22.10

32.40

42.70

61.00

101.70

101.80

101.70

76.30

78.20

80.60

85.60

90.70

70.80

82.00

86.30

87.10

77.00

85.30

78.50

95.30

75.20

72.40

85.90

82.90

94.50

$252 \quad 152.40$

22.10

22.50

22.30

32.60

43.10

61.30

101.60

102.00

101.80

76.70

78.00

81.90

84.40

92.00

83.50

87.50

74.80

77.70

81.70

89.30

91.50

69.60

78.30

77.60

87.70

85.10

$296 \quad 152.40$

22.10

22.30

22.30

31.10

40.30

58.80

79.60

79.00

74.60

72.80

78.60

74.20

$-1.00$

68.80

71.10

77.10

$\begin{array}{llll}296 & 152.4 \quad 45\end{array}$

22.40

22.70

22.40

31.30

40.60

58.90

77.90

74.40

79.10

72.00

79.70

82.60

101.40

81.40

80.80

87.40

85.00

101.80

101.50

90.10

84.90

88.40

81.30

$-1.00 \quad 69.40$

68.20

69.40

$342 \quad 152.4 \quad 45$

22.50

22.40

22.40

30.90

39.80

58.80

76.00

77.80

73.10

78.10

69.20

80.40

101.50

84.40

82.50

75.00

80.80

102.00

101.70

91.20

85.50

91.70

91.60

$\begin{array}{rr}7.00 & 69.40 \\ -1.00 & 67.50\end{array}$

66.60

67.10

101.40

101.70

101.70

82.90

89.90

80.80

84.30

73.40

90.80

80.00

89.40

$342 \quad 152.4 \quad 0$

22.50

22.40

22.40

30.50

39.10

57.70

101.40

101.70

101.40

71.60

69.40

72.70

76.90

78.30

85.60

70.80

76.40

79.50

82.60

66.70

70.70

75.20

85.00

87.30

$-1.00$

65.50

62.80

73.60

71.00

82.00

78.30 
$\begin{array}{llll}387 & 152.4 \quad 0\end{array}$

22.60

22.50

70.20

22.40

30.00

68.60

$-1.00$

67.20

$-1.00$

64.40

65.50

$-1.00$

64.40

$\begin{array}{lll}387 & 152.4 \quad 45\end{array}$

22.80

22.80

69.30

22.50

61.60

38.40
71.70
69.90
69.50
71.80

56.90

101.40

101.80

101.70

76.20

77.60

84.90

75.70

78.50

81.70

73.70

84.00

86.20

82.00

77.10

$-1.00$

29.80

$37.80 \quad 56.50$

71.00

72.90

69.70

74.80

65.00

$-1.00$

66.00

64.40

65.50

$-1.00$

63.80

$\begin{array}{lll}427 & 152.4 \quad 45\end{array}$

23.00

22.80

22.50

65.00

75.00

63.50

77.30

101.40

101.80

101.50

$80.60 \quad 87.80$

76.90

81.60

70.80

89.00

77.50

85.60

67.50

29.50

37.20

55.80

69.90

72.00

68.40

$-1.00$

64.60

$-1.00$

62.60

$-1.00$

58.60

$\begin{array}{llll}427 & 152.4 \quad 0\end{array}$

23.20

23.00

22.80

67.00

62.40

$-1.00$

65.20

$-1.00$

61.00

$-1.00$

60.10

$\begin{array}{llll}467 & 152.4 \quad 0\end{array}$

66.40

73.90

64.40

63.70

73.60

62.50

76.30

101.40

101.80

87.60

79.70

80.60

76.20

88.70

64.00

70.00

84.90

29.50

37.00

55.60

75.50

70.50

63.50

63.20

68.40

67.80

71.90

66.10

69.40

76.70

67.90

24.40

24.60

24.60

27.80

33.40

50.90

64.40

73.30

53.90

66.20

66.30

59.00

61.80

70.30

61.90

67.30

101.40

101.80

101.50

75.70

84.00

75.80

77.00

80.10

87.60

76.60

76.20

$-1.00$

56.60

$\begin{array}{lll}467 & 152.4 \quad 45\end{array}$

24.30

24.70

24.60

28.30

33.90

52.10

70.30

67.60

71.30

58.40

$-1.00$

60.70

$-1.00$

58.80

59.30

72.10

61.70

72.60

102.50

102.90

102.50

76.00

82.30

72.60

73.50

74.50

85.80

74.60

75.20

$509152.4 \quad 45$

24.30

24.70

24.60

62.20

62.50

28.30

64.10

58.40

$-1.00$

60.90

59.50

$-1.00$

58.90

61.10

$\begin{array}{lll}509 & 152.4 \quad 0\end{array}$

24.60

24.80

64.00

24.60

28.90

65.20

61.40

57.90

102.50

102.90

102.80

76.90

87.00

75.00

78.70

$68.10 \quad 87.30$

74.00

83.40

$-1.00$

60.50

34.20

52.50

102.50

102.60

102.60

68.10

70.40

76.50

85.90

71.30

74.50

78.00

61.60

71.60

68.10

87.00

58.40

72.60

74.10

82.90

$-1.00$

58.20

60.70

35.30
66.10
66.90
64.10

53.40

101.10

101.50

101.20

72.90

75.60

80.90

73.80

75.00

70.30

76.50

82.30 


\begin{tabular}{|c|c|c|c|c|c|c|c|}
\hline $\begin{array}{l}-1.00 \\
551152.4 \\
24.60\end{array}$ & $\begin{array}{l}57.90 \\
0\end{array}$ & 56.60 & 64.60 & 67.10 & 75.70 & 74.10 & \\
\hline 24.80 & 24.60 & 28.90 & 35.00 & 53.20 & 101.10 & 101.20 & 101.20 \\
\hline 64.00 & 60.30 & 64.90 & 65.70 & 72.20 & 74.70 & 79.90 & \\
\hline-1.00 & 63.10 & 61.00 & 66.40 & 68.80 & 73.10 & 74.60 & \\
\hline-1.00 & 57.70 & 60.30 & 63.70 & 70.00 & 75.20 & 81.40 & \\
\hline-1.00 & 57.70 & 56.20 & 63.70 & 66.40 & 75.20 & 73.30 & \\
\hline $\begin{array}{l}551152.4 \\
24.60\end{array}$ & 45 & & & & & & \\
\hline 24.80 & 24.60 & 29.10 & 35.30 & 52.70 & 100.80 & 100.90 & 100.80 \\
\hline 63.20 & 61.90 & 63.10 & 67.30 & 69.20 & 72.70 & 81.70 & \\
\hline-1.00 & 59.90 & 57.50 & 64.00 & 70.20 & 74.10 & 76.40 & \\
\hline-1.00 & 58.50 & 59.40 & 62.20 & 71.60 & 67.60 & 86.60 & \\
\hline-1.00 & 55.50 & 61.40 & 58.90 & 71.90 & 73.30 & 82.20 & \\
\hline $\begin{array}{l}597152.4 \\
24.80\end{array}$ & 45 & & & & & & \\
\hline 25.10 & 24.80 & 29.00 & 34.90 & 52.60 & 100.80 & 101.10 & 100.80 \\
\hline 62.80 & 61.40 & 62.80 & 67.00 & 68.70 & 72.60 & 81.90 & \\
\hline-1.00 & 59.40 & 56.80 & 63.40 & 69.80 & 73.80 & 75.60 & \\
\hline-1.00 & 58.50 & 58.80 & 62.00 & 71.00 & 67.10 & 86.40 & \\
\hline-1.00 & 55.20 & 61.00 & 58.50 & 70.90 & 73.00 & 81.60 & \\
\hline $\begin{array}{l}597152.4 \\
24.80\end{array}$ & 0 & & & & & & \\
\hline 25.10 & 24.80 & 29.00 & 35.00 & 53.10 & 100.80 & 101.10 & 101.10 \\
\hline 62.60 & 59.30 & 63.80 & 64.70 & 72.00 & 74.70 & 80.40 & \\
\hline-1.00 & 62.00 & 59.30 & 65.50 & 66.50 & 71.60 & 72.00 & \\
\hline-1.00 & 56.40 & 59.00 & 62.00 & 68.70 & 72.90 & 80.50 & \\
\hline-1.00 & 56.40 & 55.50 & 61.50 & 66.10 & 73.90 & 72.90 & \\
\hline $\begin{array}{l}208200.0 \\
23.00\end{array}$ & 30 & & & & & & \\
\hline 22.50 & 22.50 & 33.80 & 45.90 & 66.40 & 103.20 & 103.50 & 103.20 \\
\hline 90.90 & 86.60 & 89.90 & 86.70 & 91.20 & 91.90 & 97.60 & \\
\hline-1.00 & 90.00 & 89.20 & 89.50 & 91.50 & 97.30 & 95.60 & \\
\hline-1.00 & 90.60 & 90.90 & 91.80 & 93.60 & 89.80 & 100.30 & \\
\hline-1.00 & 89.00 & 86.00 & 89.40 & 90.20 & 91.10 & 96.20 & \\
\hline $\begin{array}{l}208200.0 \\
23.20\end{array}$ & 345 & & & & & & \\
\hline 23.20 & 22.70 & 33.90 & 45.40 & 64.30 & 103.10 & 103.40 & 103.30 \\
\hline 89.10 & 87.00 & 88.70 & 87.80 & 92.80 & 92.20 & 97.90 & \\
\hline-1.00 & 88.20 & 89.30 & 87.20 & 90.50 & 96.30 & 92.30 & \\
\hline-1.00 & 88.40 & 87.80 & 89.60 & 90.70 & 89.40 & 98.60 & \\
\hline-1.00 & 84.70 & 82.90 & 85.00 & 89.60 & 90.30 & 95.10 & \\
\hline 252200.0 & 345 & & & & & & \\
\hline 23.70 & & & & & & & \\
\hline 23.70 & 23.20 & 30.70 & 40.80 & 59.50 & 100.90 & 101.10 & 101.10 \\
\hline 81.60 & 80.90 & 81.30 & 81.50 & 86.60 & 86.70 & 92.70 & \\
\hline-1.00 & 79.60 & 81.40 & 79.40 & 84.00 & 91.40 & 86.90 & \\
\hline-1.00 & 81.00 & 80.90 & 82.60 & 86.10 & 84.00 & 94.60 & \\
\hline-1.00 & 77.30 & 76.00 & 79.20 & 82.60 & 84.90 & 88.10 & \\
\hline 252200.0 & 30 & & & & & & \\
\hline 23.70 & & & & & & & \\
\hline 24.00 & 23.20 & 31.40 & 41.40 & 60.20 & 101.40 & 101.80 & 101.70 \\
\hline 82.20 & 79.40 & 82.60 & 78.20 & 86.00 & 86.00 & 93.40 & \\
\hline-1.00 & 81.90 & 78.40 & 81.50 & 84.00 & 92.00 & 88.80 & \\
\hline
\end{tabular}




\begin{tabular}{|c|c|c|c|c|c|c|c|}
\hline-1.00 & 81.20 & 82.90 & 83.50 & 85.20 & 80.90 & 95.50 & \\
\hline-1.00 & 80.70 & 77.70 & 79.20 & 85.30 & 84.40 & 92.50 & \\
\hline \multicolumn{8}{|c|}{$\begin{array}{l}296200.030 \\
23.80\end{array}$} \\
\hline 23.80 & 23.50 & 31.10 & 40.50 & 59.10 & 101.50 & 101.70 & \multirow[t]{5}{*}{101.70} \\
\hline 82.50 & 79.40 & 81.90 & 77.50 & 84.60 & 85.50 & 92.10 & \\
\hline-1.00 & 81.20 & 77.30 & 81.00 & 83.70 & 90.40 & 88.40 & \\
\hline-1.00 & 80.20 & 82.60 & 81.90 & 85.30 & 80.40 & 95.80 & \\
\hline-1.00 & 80.70 & 77.80 & 79.20 & 84.30 & 83.30 & 91.50 & \\
\hline \multicolumn{8}{|c|}{$\begin{array}{l}296 \quad 200.03 \quad 45 \\
23.80\end{array}$} \\
\hline 24.10 & 23.70 & 31.10 & 40.00 & 58.80 & 101.50 & 101.80 & \multirow[t]{5}{*}{101.70} \\
\hline 82.50 & 80.70 & 80.30 & 81.70 & 84.20 & 86.40 & 90.30 & \\
\hline-1.00 & 78.90 & 80.70 & 77.80 & 86.30 & 92.80 & 89.10 & \\
\hline-1.00 & 81.20 & 80.70 & 83.80 & 81.70 & 84.60 & 90.50 & \\
\hline-1.00 & 77.50 & 76.40 & 78.90 & 81.60 & 84.10 & 88.20 & \\
\hline \multicolumn{8}{|c|}{$342200.03 \quad 45$} \\
\hline 24.10 & 23.80 & 30.80 & 39.10 & 58.50 & 101.50 & 101.70 & \multirow[t]{5}{*}{101.70} \\
\hline 81.30 & 79.60 & 79.40 & 80.60 & 83.20 & 85.50 & 89.40 & \\
\hline-1.00 & 77.60 & 79.60 & 76.70 & 85.20 & 92.20 & 87.70 & \\
\hline-1.00 & 79.90 & 79.40 & 82.90 & 80.50 & 83.20 & 89.10 & \\
\hline-1.00 & 76.40 & 75.30 & 77.90 & 80.30 & 83.40 & 87.00 & \\
\hline \multicolumn{8}{|c|}{342200.030} \\
\hline \multicolumn{8}{|c|}{24.10} \\
\hline 24.40 & 24.10 & 31.10 & 39.70 & 59.00 & 101.50 & 102.00 & \multirow[t]{5}{*}{101.70} \\
\hline 81.10 & 77.60 & 79.90 & 76.70 & 81.90 & 86.10 & 92.00 & \\
\hline-1.00 & 79.70 & 75.30 & 80.80 & 82.90 & 87.00 & 88.00 & \\
\hline-1.00 & 77.90 & 80.50 & 80.90 & 85.30 & 79.70 & 94.90 & \\
\hline-1.00 & 79.00 & 75.80 & 79.00 & 81.70 & 82.90 & 88.90 & \\
\hline \multicolumn{8}{|c|}{387200.030} \\
\hline 24.30 & & & & & & & \\
\hline 24.80 & 24.60 & 30.80 & 38.60 & 56.90 & 99.60 & 99.90 & \multirow[t]{5}{*}{99.80} \\
\hline 77.30 & 73.00 & 75.80 & 71.90 & 78.50 & 81.60 & 89.40 & \\
\hline-1.00 & 76.10 & 74.40 & 77.20 & 79.20 & 84.30 & 83.80 & \\
\hline-1.00 & 75.80 & 77.60 & 78.40 & 82.90 & 75.60 & 92.90 & \\
\hline-1.00 & 74.40 & 71.60 & 73.70 & 79.10 & 77.50 & 86.20 & \\
\hline \multicolumn{8}{|c|}{387200.0345} \\
\hline 24.80 & 24.60 & 30.80 & 38.50 & 56.70 & 99.90 & 99.90 & \multirow[t]{5}{*}{99.90} \\
\hline 77.30 & 75.30 & 76.10 & 75.80 & 80.70 & 82.50 & 89.20 & \\
\hline-1.00 & 75.30 & 77.20 & 75.10 & 80.40 & 87.30 & 83.40 & \\
\hline-1.00 & 75.80 & 75.30 & 78.40 & 79.70 & 79.70 & 84.80 & \\
\hline-1.00 & 71.90 & 70.70 & 73.90 & 75.10 & 79.40 & 80.50 & \\
\hline \multicolumn{8}{|c|}{$427 \quad 200.03 \quad 45$} \\
\hline 24.80 & 24.60 & 30.60 & 37.80 & 56.00 & 99.90 & 99.90 & \multirow[t]{5}{*}{99.90} \\
\hline 77.00 & 74.90 & 75.30 & 74.90 & 80.20 & 82.00 & 88.90 & \\
\hline-1.00 & 74.90 & 76.70 & 74.70 & 80.20 & 86.70 & 82.90 & \\
\hline-1.00 & 75.30 & 74.60 & 77.60 & 79.00 & 79.30 & 84.20 & \\
\hline-1.00 & 71.40 & 70.10 & 73.50 & 74.60 & 78.60 & 79.70 & \\
\hline \multicolumn{8}{|c|}{427200.030} \\
\hline 25.10 & 24.60 & 30.40 & 37.70 & 55.70 & 100.20 & 100.20 & \multirow[t]{2}{*}{100.20} \\
\hline 76.60 & 72.10 & 74.70 & 70.50 & 77.30 & 80.20 & 86.10 & \\
\hline
\end{tabular}




\begin{tabular}{|c|c|c|c|c|c|c|c|}
\hline-1.00 & 74.70 & 73.40 & 76.10 & 77.90 & 84.10 & 82.50 & \\
\hline-1.00 & 74.70 & 76.50 & 77.00 & 81.90 & 74.30 & 93.80 & \\
\hline-1.00 & 73.30 & 70.80 & 73.30 & 78.40 & 76.00 & 85.50 & \\
\hline \multicolumn{8}{|c|}{467200.030} \\
\hline 25.20 & 24.90 & 30.30 & 37.00 & 55.40 & 100.30 & 100.30 & 100.30 \\
\hline 75.80 & 71.70 & 74.30 & 70.00 & 76.10 & 79.90 & 85.30 & \\
\hline-1.00 & 74.30 & 72.90 & 75.30 & 77.60 & 83.50 & 82.10 & \\
\hline-1.00 & 74.00 & 75.90 & 76.60 & 81.10 & 73.90 & 93.20 & \\
\hline-1.00 & 72.90 & 70.30 & 72.30 & 77.90 & 75.80 & 85.10 & \\
\hline \multicolumn{8}{|c|}{$\begin{array}{l}467 \quad 200.0345 \\
24.70\end{array}$} \\
\hline 25.20 & 24.90 & 30.30 & 37.30 & 55.30 & 99.60 & 99.80 & 99.60 \\
\hline 75.80 & 72.90 & 73.40 & 73.90 & 77.60 & 80.20 & 86.90 & \\
\hline-1.00 & 72.50 & 74.30 & 72.50 & 79.90 & 85.80 & 83.10 & \\
\hline-1.00 & 73.40 & 71.60 & 77.00 & 73.20 & 79.30 & 77.70 & \\
\hline-1.00 & 69.20 & 68.30 & 71.80 & 72.50 & 78.40 & 79.40 & \\
\hline \multicolumn{8}{|c|}{$\begin{array}{l}509200.03 \quad 45 \\
24.80\end{array}$} \\
\hline 25.20 & 24.90 & 29.90 & 36.20 & 54.10 & 100.30 & 100.30 & 100.30 \\
\hline 74.40 & 72.60 & 72.80 & 73.40 & 77.00 & 79.60 & 86.40 & \\
\hline-1.00 & 71.90 & 73.80 & 72.10 & 78.80 & 84.50 & 82.20 & \\
\hline-1.00 & 72.40 & 70.70 & 76.50 & 72.60 & 78.80 & 76.30 & \\
\hline-1.00 & 68.70 & 67.80 & 71.40 & 72.40 & 77.00 & 78.80 & \\
\hline \multicolumn{8}{|c|}{$\begin{array}{l}509200.030 \\
24.80\end{array}$} \\
\hline 25.20 & 24.90 & 29.90 & 36.30 & 54.30 & 99.40 & 99.90 & 99.50 \\
\hline 74.40 & 70.20 & 72.50 & 68.40 & 73.40 & 79.10 & 82.50 & \\
\hline-1.00 & 72.60 & 71.20 & 74.20 & 76.30 & 81.40 & 80.80 & \\
\hline-1.00 & 72.10 & 74.00 & 75.10 & 79.30 & 73.10 & 91.70 & \\
\hline-1.00 & 71.20 & 68.50 & 71.60 & 76.10 & 74.00 & 83.30 & \\
\hline \multicolumn{8}{|c|}{$\begin{array}{l}551200.030 \\
24.40\end{array}$} \\
\hline 24.10 & 24.30 & 27.70 & 34.10 & 52.70 & 100.90 & 100.90 & 100.90 \\
\hline 73.10 & 68.30 & 71.20 & 66.90 & 73.70 & 78.50 & 83.30 & \\
\hline-1.00 & 71.50 & 71.20 & 73.20 & 76.40 & 81.10 & 81.10 & \\
\hline-1.00 & 72.30 & 73.40 & 75.20 & 80.70 & 71.00 & 92.70 & \\
\hline-1.00 & 68.80 & 66.40 & 69.10 & 75.30 & 73.50 & 82.80 & \\
\hline \multirow{2}{*}{\multicolumn{8}{|c|}{$\begin{array}{l}551200.0345 \\
24.80\end{array}$}} \\
\hline & & & & & & & \\
\hline 24.10 & 24.30 & 27.30 & 33.80 & 52.40 & 100.90 & 101.20 & 101.20 \\
\hline 73.10 & 71.00 & 72.00 & 70.90 & 79.00 & 80.00 & 88.40 & \\
\hline-1.00 & 71.50 & 74.20 & 72.10 & 78.10 & 83.70 & 80.80 & \\
\hline-1.00 & 71.20 & 69.50 & 74.30 & 76.80 & 75.80 & 82.20 & \\
\hline-1.00 & 66.70 & 65.50 & 69.10 & 71.80 & 74.30 & 77.20 & \\
\hline \multicolumn{8}{|c|}{$\begin{array}{l}597200.03 \quad 45 \\
24.60\end{array}$} \\
\hline 24.10 & 24.10 & 25.60 & 32.10 & 50.50 & 100.90 & 101.10 & 101.10 \\
\hline 72.50 & 70.20 & 70.80 & 69.60 & 77.80 & 78.80 & 87.10 & \\
\hline-1.00 & 70.50 & 73.10 & 71.00 & 76.90 & 82.50 & 76.80 & \\
\hline-1.00 & 69.90 & 68.20 & 73.10 & 75.60 & 74.70 & 80.80 & \\
\hline-1.00 & 65.80 & 64.60 & 68.40 & 70.60 & 73.00 & 76.00 & \\
\hline \multicolumn{8}{|c|}{$\begin{array}{l}597200.030 \\
24.80\end{array}$} \\
\hline 23.90 & 24.10 & 26.20 & 32.30 & 51.00 & 101.00 & 101.30 & 101.10 \\
\hline
\end{tabular}


72.30

$-1.00$

$-1.00$

$-1.00$
68.30

70.80

71.60

67.60
71.10

70.80

72.20

65.40
66.00

72.00

74.50

64.60
75.40

75.40

78.00

75.90
77.30

82.00

68.40

73.80
87.90

80.00

87.30

82.50 
$50.8 \mathrm{~mm}$ column, $28.2 \mathrm{~mm}$ polystyrene spheres

\section{Format}

First 4 lines:

\# radial profiles \# radial T/C positions \# wall temperatures \# angular positions

Radial $\mathrm{T} / \mathrm{C}$ positions in $\mathrm{mm}$ from center of bed

Axial $\mathrm{T} / \mathrm{C}$ positions on tube wall (-ve means calming section) in $\mathrm{mm}$

Tube radius Sphere diameter

Data then follow in blocks:

Re L (mm) Angle

$\mathrm{T}_{\text {in }}$ (entrance to calming section)

Tube wall temperature measurements

Radial profiles (-1 means no temperature measured; note temperatures at $r=0.0$ were not used)

\begin{tabular}{|c|c|c|c|c|c|c|c|}
\hline 4 & 8 & 2 & & & & & \\
\hline 0.0 & 7.6 & 11.6 & 14.2 & 17.8 & 20.4 & 23.2 & \\
\hline-127.0 & -76.2 & -25.4 & -15.9 & -6.35 & 76.2 & 228.6 & 381.0 \\
\hline 25.4 & 28.6 & & & & & & \\
\hline $420 \quad 10^{\circ}$ & 50 & & & & & & \\
\hline 22.40 & & & & & & & \\
\hline 23.50 & 25.30 & 42.40 & 52.60 & 71.40 & 101.20 & 101.40 & 101.10 \\
\hline 36.80 & 36.40 & 36.90 & 41.40 & 44.30 & 50.40 & 76.70 & \\
\hline-1.00 & 33.20 & 40.70 & 32.80 & 60.00 & 54.30 & 80.60 & \\
\hline-1.00 & 37.40 & 37.30 & 41.00 & 37.70 & 60.40 & 71.80 & \\
\hline-1.00 & 33.40 & 38.80 & 32.60 & 60.70 & 53.10 & 92.50 & \\
\hline $420 \quad 10^{\circ}$ & 545 & & & & & & \\
\hline 22.50 & & & & & & & \\
\hline 23.70 & 25.60 & 42.40 & 52.60 & 71.60 & 101.40 & 101.40 & 101.20 \\
\hline 36.80 & 36.60 & 39.30 & 52.10 & 46.50 & 57.40 & 73.10 & \\
\hline-1.00 & 34.90 & 32.20 & 36.90 & 39.50 & 76.30 & 68.70 & \\
\hline-1.00 & 37.70 & 39.70 & 43.40 & 44.10 & 54.90 & 67.10 & \\
\hline-1.00 & 36.40 & 31.30 & 37.40 & 43.70 & 80.10 & 84.90 & \\
\hline $48510^{\circ}$ & 545 & & & & & & \\
\hline 22.60 & & & & & & & \\
\hline 23.80 & 25.70 & 42.30 & 52.40 & 71.50 & 101.40 & 101.40 & 101.20 \\
\hline 37.50 & 37.90 & 39.70 & 51.60 & 47.10 & 55.40 & 72.40 & \\
\hline-1.00 & 36.10 & 31.70 & 35.70 & 39.70 & 71.20 & 68.20 & \\
\hline-1.00 & 38.00 & 39.40 & 44.50 & 43.10 & 54.30 & 64.70 & \\
\hline-1.00 & 36.60 & 31.10 & 36.70 & 42.80 & 79.30 & 84.00 & \\
\hline $48510^{\prime}$ & 50 & & & & & & \\
\hline 22.70 & & & & & & & \\
\hline 23.80 & 25.60 & 42.00 & 52.00 & 71.00 & 101.30 & 101.40 & 101.20 \\
\hline 36.90 & 31.80 & 43.00 & 37.70 & 63.80 & 69.00 & 81.40 & \\
\hline-1.00 & 37.00 & 29.50 & 51.60 & 36.50 & 68.10 & 65.00 & \\
\hline-1.00 & 37.00 & 38.90 & 43.30 & 42.70 & 75.80 & 60.80 & \\
\hline-1.00 & 36.10 & 32.40 & 47.10 & 37.80 & 70.60 & 82.20 & \\
\hline $\begin{array}{lll}530 & 10^{\circ}\end{array}$ & 50 & & & & & & \\
\hline 22.60 & & & & & & & \\
\hline 23.80 & 25.70 & 41.30 & 51.30 & 70.10 & 101.20 & 101.40 & 101.20 \\
\hline 36.70 & 31.80 & 44.20 & 36.50 & 65.40 & 66.60 & 80.30 & \\
\hline
\end{tabular}




\begin{tabular}{|c|c|c|c|c|c|c|c|}
\hline-1.00 & 36.60 & 29.10 & 51.80 & 35.80 & 64.20 & 63.40 & \\
\hline-1.00 & 36.60 & 37.50 & 42.20 & 40.30 & 71.40 & 55.80 & \\
\hline-1.00 & 35.60 & 31.60 & 45.00 & 37.00 & 68.10 & 80.80 & \\
\hline \multicolumn{8}{|c|}{$\begin{array}{lll}530 & 107.95 \quad 45\end{array}$} \\
\hline 23.90 & 25.70 & 41.80 & 51.60 & 70.80 & 101.50 & 101.40 & 101.10 \\
\hline 36.20 & 38.50 & 44.70 & 52.30 & 56.40 & 51.00 & 80.40 & \\
\hline-1.00 & 32.60 & 36.20 & 30.50 & 49.60 & 52.80 & 78.60 & \\
\hline-1.00 & 36.10 & 38.20 & 39.40 & 51.90 & 49.10 & 84.00 & \\
\hline-1.00 & 34.50 & 34.00 & 31.20 & 50.20 & 63.20 & 86.90 & \\
\hline \multicolumn{8}{|c|}{$\begin{array}{l}575 \quad 107.95 \quad 45 \\
23.00\end{array}$} \\
\hline 23.00 & 23.40 & 37.50 & 48.00 & 67.60 & 100.00 & 100.00 & 99.90 \\
\hline 34.30 & 36.40 & 41.20 & 45.40 & 59.90 & 46.90 & 82.90 & \\
\hline-1.00 & 31.80 & 34.20 & 29.50 & 25.60 & 57.90 & 75.90 & \\
\hline-1.00 & 35.10 & 36.00 & 39.30 & 48.20 & 49.10 & 82.80 & \\
\hline-1.00 & 33.50 & 32.10 & 30.00 & 47.40 & 60.20 & 84.90 & \\
\hline \multicolumn{8}{|c|}{575107.950} \\
\hline 23.40 & 24.10 & 38.10 & 48.40 & 68.10 & 99.90 & 99.90 & 99.90 \\
\hline 35.80 & 38.00 & 29.90 & 48.50 & 39.70 & 62.80 & 77.30 & \\
\hline-1.00 & 32.90 & 38.90 & 36.70 & 56.60 & 68.30 & 77.60 & \\
\hline-1.00 & 34.00 & 33.10 & 38.30 & 31.90 & 58.00 & 57.70 & \\
\hline-1.00 & 32.10 & 38.60 & 35.20 & 54.60 & 66.40 & 85.50 & \\
\hline \multicolumn{8}{|c|}{617107.950} \\
\hline 23.50 & 24.10 & 38.70 & 48.90 & 68.10 & 99.90 & 99.90 & 99.90 \\
\hline 34.30 & 34.80 & 35.00 & 38.40 & 38.60 & 46.90 & 70.70 & \\
\hline-1.00 & 31.00 & 37.30 & 31.80 & 57.20 & 44.60 & 72.50 & \\
\hline-1.00 & 32.90 & 34.10 & 35.50 & 33.50 & 55.40 & 65.00 & \\
\hline-1.00 & 31.80 & 37.80 & 31.60 & 54.30 & 47.20 & 88.00 & \\
\hline \multicolumn{8}{|c|}{$\begin{array}{l}617 \quad 107.95 \quad 45 \\
23.50\end{array}$} \\
\hline 23.80 & 24.70 & 39.20 & 49.50 & 68.40 & 100.00 & 100.00 & 100.00 \\
\hline 35.20 & 35.60 & 38.10 & 45.30 & 44.70 & 48.80 & 67.80 & \\
\hline-1.00 & 36.20 & 29.70 & 35.60 & 36.70 & 64.60 & 62.60 & \\
\hline-1.00 & 35.60 & 35.50 & 46.40 & 38.00 & 49.20 & 59.00 & \\
\hline-1.00 & 33.90 & 30.20 & 34.70 & 40.20 & 70.50 & 81.70 & \\
\hline \multirow{2}{*}{\multicolumn{8}{|c|}{23.70}} \\
\hline & & & & & & & \\
\hline $\begin{array}{l}23.90 \\
35.40\end{array}$ & 24.80 & 39.10 & 49.60 & 68.40 & 100.00 & 100.00 & 100.00 \\
\hline 35.40 & 36.60 & 38.30 & 44.50 & 44.70 & 48.40 & 67.20 & \\
\hline-1.00 & 36.10 & 29.70 & 35.50 & 36.70 & 62.80 & 62.10 & \\
\hline-1.00 & 35.30 & 35.30 & 47.20 & 37.70 & 48.70 & 55.90 & \\
\hline-1.00 & 33.90 & 30.20 & 34.70 & 39.90 & 69.20 & 80.90 & \\
\hline 66010 & 50 & & & & & & \\
\hline \multicolumn{8}{|c|}{23.70} \\
\hline 23.90 & 24.80 & 39.80 & 49.60 & 68.40 & 99.90 & 99.80 & 99.70 \\
\hline 34.20 & 34.80 & 35.20 & 38.00 & 38.10 & 46.40 & 70.70 & \\
\hline-1.00 & 31.00 & 36.80 & 31.90 & 56.60 & 42.90 & 71.30 & \\
\hline-1.00 & 32.70 & 34.00 & 34.90 & 33.70 & 54.40 & 64.20 & \\
\hline-1.00 & 31.50 & 39.10 & 31.80 & 54.80 & 45.90 & 86.70 & \\
\hline \multicolumn{8}{|c|}{$\begin{array}{l}705107.950 \\
23.70\end{array}$} \\
\hline 23.90 & 25.00 & 39.80 & 49.60 & 68.10 & 99.70 & 99.80 & 99.70 \\
\hline & & & & 21 & & & \\
\hline
\end{tabular}




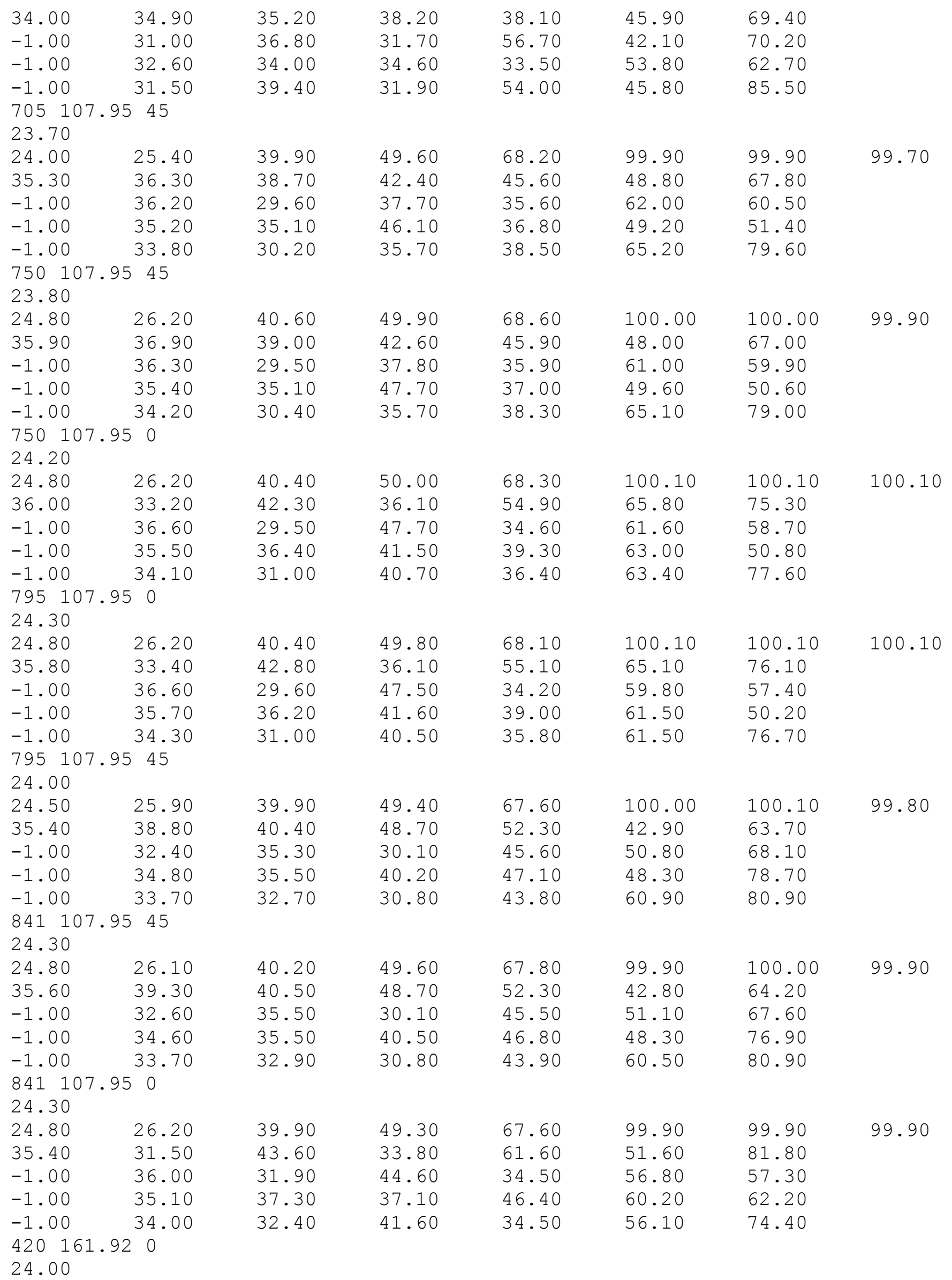




\begin{tabular}{|c|c|c|c|c|c|c|c|}
\hline 24.90 & 26.50 & 44.70 & 53.40 & 70.90 & 101.40 & 101.40 & 101.10 \\
\hline 53.70 & 52.00 & 63.20 & 52.80 & 67.10 & 61.70 & 84.60 & \\
\hline-1.00 & 52.50 & 52.20 & 55.70 & 58.10 & 74.70 & 75.80 & \\
\hline-1.00 & 46.90 & 51.70 & 55.80 & 59.90 & 80.10 & 75.80 & \\
\hline-1.00 & 56.00 & 57.80 & 64.90 & 70.50 & 66.40 & 93.20 & \\
\hline \multicolumn{8}{|c|}{$\begin{array}{l}420161.9245 \\
24.00\end{array}$} \\
\hline 24.90 & 26.40 & 44.70 & 53.80 & 71.40 & 101.20 & 101.40 & \multirow[t]{5}{*}{101.10} \\
\hline 53.80 & 56.00 & 54.60 & 66.10 & 59.10 & 63.80 & 77.20 & \\
\hline-1.00 & 51.30 & 48.50 & 54.10 & 66.20 & 78.10 & 84.20 & \\
\hline-1.00 & 52.30 & 60.40 & 55.40 & 72.60 & 78.20 & 86.90 & \\
\hline-1.00 & 52.90 & 53.10 & 61.50 & 60.00 & 68.90 & 90.60 & \\
\hline \multicolumn{8}{|c|}{$\begin{array}{l}485161.92 \quad 45 \\
24.00\end{array}$} \\
\hline 24.80 & 26.40 & 44.70 & 53.10 & 70.50 & 101.10 & 101.10 & \multirow[t]{5}{*}{101.10} \\
\hline 53.40 & 56.60 & 54.00 & 65.20 & 58.70 & 61.60 & 76.20 & \\
\hline-1.00 & 50.80 & 47.70 & 52.80 & 63.60 & 75.80 & 81.40 & \\
\hline-1.00 & 51.60 & 58.90 & 56.30 & 70.20 & 76.70 & 86.50 & \\
\hline-1.00 & 52.80 & 52.20 & 61.80 & 57.70 & 67.90 & 87.40 & \\
\hline \multicolumn{8}{|c|}{485161.920} \\
\hline 24.00 & & & & & & & \\
\hline 24.60 & 26.40 & 44.20 & 52.40 & 69.60 & 100.00 & 100.00 & \multirow[t]{5}{*}{99.80} \\
\hline 53.00 & 49.70 & 62.30 & 52.80 & 69.60 & 58.20 & 83.70 & \\
\hline-1.00 & 51.60 & 52.50 & 53.70 & 54.70 & 72.00 & 69.80 & \\
\hline-1.00 & 45.80 & 50.50 & 55.50 & 62.60 & 71.10 & 80.80 & \\
\hline-1.00 & 54.90 & 55.40 & 63.30 & 64.70 & 63.20 & 89.50 & \\
\hline \multicolumn{8}{|c|}{530161.920} \\
\hline 24.10 & & & & & & & \\
\hline 24.90 & 26.50 & 44.20 & 52.30 & 69.50 & 100.60 & 100.60 & \multirow[t]{5}{*}{100.60} \\
\hline 53.20 & 50.20 & 62.30 & 52.90 & 69.50 & 58.40 & 84.40 & \\
\hline-1.00 & 51.60 & 52.60 & 53.80 & 55.10 & 71.60 & 69.80 & \\
\hline-1.00 & 45.90 & 51.10 & 54.30 & 63.30 & 71.80 & 80.90 & \\
\hline-1.00 & 54.60 & 55.70 & 63.20 & 64.90 & 63.40 & 90.30 & \\
\hline \multirow{2}{*}{\multicolumn{8}{|c|}{$530 \quad 161.92 \quad 45$}} \\
\hline & & & & & & & \\
\hline 25.10 & 26.50 & 44.20 & 52.40 & 69.60 & 100.60 & 100.60 & \multirow[t]{5}{*}{100.60} \\
\hline 52.60 & 56.40 & 53.90 & 64.40 & 58.70 & 61.80 & 74.40 & \\
\hline-1.00 & 50.20 & 46.90 & 51.60 & 61.10 & 70.60 & 78.80 & \\
\hline-1.00 & 51.30 & 57.90 & 55.30 & 70.40 & 76.30 & 87.90 & \\
\hline-1.00 & 52.00 & 52.00 & 61.30 & 55.40 & 66.20 & 84.70 & \\
\hline \multicolumn{8}{|c|}{$\begin{array}{l}575161.9245 \\
24.10\end{array}$} \\
\hline 24.90 & 26.50 & 43.60 & 51.40 & 68.80 & 100.60 & 100.60 & \multirow[t]{7}{*}{100.60} \\
\hline 51.90 & 56.10 & 53.40 & 63.70 & 58.20 & 61.20 & 73.40 & \\
\hline-1.00 & 49.80 & 46.70 & 50.80 & 59.90 & 69.50 & 77.00 & \\
\hline-1.00 & 50.80 & 56.90 & 55.40 & 68.80 & 75.10 & 86.10 & \\
\hline-1.00 & 51.90 & 52.00 & 61.10 & 55.00 & 65.50 & 83.10 & \\
\hline \multirow{2}{*}{\multicolumn{7}{|c|}{$\begin{array}{l}575161.920 \\
24.10\end{array}$}} & \\
\hline & & & & & & & \\
\hline 24.90 & 26.50 & 43.60 & 51.60 & 69.00 & 100.60 & 100.60 & \multirow[t]{5}{*}{100.60} \\
\hline 52.50 & 50.10 & 62.30 & 51.20 & 67.10 & 59.00 & 84.00 & \\
\hline-1.00 & 51.20 & 51.70 & 53.80 & 54.30 & 69.10 & 69.70 & \\
\hline-1.00 & 46.00 & 51.60 & 53.40 & 60.90 & 73.90 & 75.40 & \\
\hline-1.00 & 53.90 & 55.60 & 61.90 & 66.30 & 61.40 & 90.00 & \\
\hline
\end{tabular}


22.90

22.90

50.40

22.90

36.20

60.70

45.70

66.00

102.00

102.00

102.00

$-1.00$

49.40

$-1.00$

48.90

50.30

$-1.00$

44.50

49.70

65.80

57.90

84.60

51.60

52.10

65.70

67.90

50.10

72.20

73.20

53.80

59.20

59.00

89.70

$\begin{array}{lll}617 & 161.92 \quad 45\end{array}$

22.90

22.90

37.70

47.00

66.40

61.60

50.00

23.20

50.80

50.50

$-1.00$

48.40

45.20

$-1.00$

48.50

55.00

$-1.00$

50.40

54.40

50.10

56.50

54.10

58.50

64.60

54.70

101.70

101.70

101.70

56.30

69.30

72.20

76.10

70.80

73.80

67.90

82.20

$\begin{array}{ll}660 & 161 \\ 23.00 & 0\end{array}$

22.90

23.40

38.10

47.40

66.70

101.80

101.80

101.70

50.80

50.00

48.50

61.60

54.10

56.10

68.40

50.50

57.80

71.10

75.40

54.70

$-1.00$

48.50

$-1.00$

50.40

49.80

63.70

70.20

72.30

56.00

54.50

66.50

81.10

23.50

23.70

24.40

40.20

48.70

67.20

68.10

51.50

51.60

$-1.00$

49.70

60.40

52.00

50.40

50.80

$-1.00$

45.00

59.30

59.00

61.00

101.10

101.10

101.00

57.20

86.40

65.70

65.50

68.40

73.70

59.30

87.70

$705 \quad 161.920$

23.70

23.90

24.70

40.20

48.70

67.00

55.90

59.40

48.30

53.90

$-1.00$

46.10

51.50

52.40

53.00

52.40

$-1.00$

50.10

57.90

54.40

$\begin{array}{lll}705 & 161.92 \quad 45\end{array}$

23.70

23.90

24.80

40.20

48.70

51.50

52.60

$-1.00$

48.30

58.20

47.20

50.80

49.00

$-1.00$

45.80

53.70

55.40

$\begin{array}{lll}750 & 161.92 \quad 45\end{array}$

23.70

23.90

24.80

40.20

48.70

51.50

57.80

53.00

$-1.00$

48.70

47.10

48.70

$-1.00$

46.10

50.90

54.50

67.00

62.40

52.30

56.10

62.30

101.10

101.10

101.10

61.90

79.20

57.80

69.60

66.30

73.50

58.80

88.10

$750 \quad 161.92 \quad 0$

23.70

23.90

24.80

52.90

67.00

61.30

52.00

55.50

61.80

101.40

101.40

101.40

63.40

74.80

63.10

68.50

71.60

65.90

60.40

87.50

49.60

40.20

48.70

67.00

60.70

101.10

101.10

101.10

62.20

73.40

61.70

67.70

71.00

65.20

60.60

86.70

$-1.00$

53.10

51.60

58.50

54.10

51.90

55.40

55.70

57.60

101.40

101.40

101.10

59.50

76.00

$58.70 \quad 70.20$

$68.10 \quad 76.40$

55.20

59.90

81.90 
$\begin{array}{lll}795 & 161.92 \quad 0\end{array}$

23.80

23.90

49.30

24.80

40.20

50.90

$-1.00$

52.50

45.50

$-1.00$

46.30

52.30

$-1.00$

49.10

$\begin{array}{lll}795 & 161.92 \quad 45\end{array}$

54.50

48.40

66.80

57.50

59.70

47.20

53.80

51.50

54.70

54.40

57.40

$\begin{array}{ll}101.40 & 101.40 \\ 58.50 & 74.70 \\ 58.10 & 68.60 \\ 66.70 & 75.60 \\ 58.90 & 81.30\end{array}$

101.40

23.70

23.90

49.50

24.80

40.20

48.40

56.90

50.80

$-1.00$

50.50

46.90

$-1.00$

48.00

50.20

$-1.00$

45.70

52.20

48.70

52.30

54.80

$841 \quad 161.92 \quad 45$

23.90

24.10

25.00

40.20

48.40

51.00

49.30

50.50

56.40

51.70

$-1.00$

48.00

46.60

50.00

$-1.00$

45.70

48.70

51.90

54.00

66.70

60.40

51.60

54.70

60.70

101.20
61.30
60.80
70.20
59.60

101.40

101.10

71.60

66.90

65.20

86.40

841161.920

23.90

24.40

25.30

40.20

48.70

56.90

49.30

52.30

50.70

45.50

$-1.00$

46.20

52.20

$-1.00$

49.00

47.20

51.30

54.80

54.40

$420 \quad 196.850$

24.00

25.00

26.20

43.00

57.70

56.40

57.30

$-1.00$

54.50

$-1.00$

56.70

$-1.00$

55.60

52.20

59.00

58.20

66.70

60.40

51.40

54.10

60.30

101.20

61.40

60.70

69.50

59.20

101.40

101.20

70.70

66.00

64.00

85.50

$420 \quad 196.85 \quad 45$

24.00

25.00

26.20

57.20

43.50

60.50

54.90

$-1.00$

56.40

$-1.00$

53.40

58.30

66.70

59.10

53.10

54.00

58.00

101.50

58.20

101.70

101.40

57.80

74.70

66.90

66.70

58.70

74.00

82.60

$-1.00 \quad 57.40$

62.20

51.90

70.20

102.20

102.20

102.20

58.20

69.30

75.60

89.40

61.20

63.70

80.40

57.70

65.40

75.70

86.80

61.10

69.40

63.50

95.00

$\begin{array}{lll}485 & 196.85 \quad 45\end{array}$

24.00

24.80

26.20

43.50

52.30

70.20

66.50

102.10

102.10

101.90

58.30

70.30

83.20

87.00

62.90

59.20

68.60

86.20

82.10

70.50

63.40

66.90

71.70

91.90

57.00

58.00

60.50

52.00

70.20

66.00

101.90

101.90

101.90

59.20

68.50

81.30

84.40

65.50

84.40

55.10

60.10

81.10

69.40

58.10

62.20

66.70

69.40

91.20

$485 \quad 196.850$

23.90

24.80

26.20

43.50

52.30

70.20

102.20

102.20

102.10

57.30

58.10

59.40

68.10

75.60

87.80

52.90

62.30

61.20

65.10

79.00

67.00

75.10

88.20 


\begin{tabular}{|c|c|c|c|c|c|c|c|}
\hline \multicolumn{8}{|c|}{$\begin{array}{l}-1.00 \quad 56.40 \\
530196.850 \\
24.00\end{array}$} \\
\hline 24.90 & 26.40 & 43.60 & 52.00 & 69.80 & 102.00 & 102.00 & 102.00 \\
\hline 56.70 & 56.90 & 56.80 & 58.00 & 65.70 & 74.40 & 85.90 & \\
\hline-1.00 & 54.10 & 52.70 & 61.00 & 61.30 & 64.50 & 77.20 & \\
\hline-1.00 & 57.10 & 59.40 & 57.80 & 64.90 & 72.80 & 87.00 & \\
\hline-1.00 & 55.20 & 58.00 & 60.80 & 65.00 & 62.20 & 88.10 & \\
\hline \multicolumn{8}{|c|}{$\begin{array}{ll}530 & 196.85 \quad 45 \\
24 & 00\end{array}$} \\
\hline 24.90 & 26.40 & 43.80 & 52.20 & 70.00 & 102.00 & 102.00 & 102.00 \\
\hline 57.20 & 57.60 & 60.20 & 60.00 & 66.10 & 77.00 & 81.10 & \\
\hline-1.00 & 55.70 & 54.60 & 59.90 & 67.60 & 65.70 & 83.50 & \\
\hline-1.00 & 52.80 & 58.30 & 55.00 & 60.60 & 78.20 & 68.90 & \\
\hline-1.00 & 57.80 & 60.00 & 59.60 & 65.00 & 71.00 & 91.10 & \\
\hline \multicolumn{8}{|c|}{$575 \quad 196.85 \quad 45$} \\
\hline 24.90 & 26.40 & 43.60 & 52.00 & 69.70 & 102.00 & 102.00 & 102.00 \\
\hline 56.90 & 57.10 & 59.70 & 60.00 & 65.20 & 74.80 & 79.30 & \\
\hline-1.00 & 55.20 & 54.10 & 59.10 & 66.30 & 65.00 & 81.80 & \\
\hline-1.00 & 52.20 & 57.60 & 55.30 & 60.40 & 76.40 & 68.30 & \\
\hline-1.00 & 57.70 & 59.30 & 58.30 & 63.80 & 69.80 & 89.70 & \\
\hline \multicolumn{8}{|c|}{$\begin{array}{l}575196.850 \\
24.00\end{array}$} \\
\hline 24.90 & 26.40 & 43.30 & 51.20 & 69.60 & 102.00 & 102.00 & 102.00 \\
\hline 56.30 & 56.40 & 56.00 & 57.40 & 61.70 & 73.00 & 82.30 & \\
\hline-1.00 & 53.70 & 52.80 & 60.50 & 62.40 & 64.50 & 77.90 & \\
\hline-1.00 & 57.40 & 58.30 & 58.00 & 61.60 & 70.50 & 85.10 & \\
\hline-1.00 & 54.80 & 57.90 & 59.40 & 66.70 & 61.30 & 87.80 & \\
\hline \multicolumn{8}{|c|}{$\begin{array}{l}617196.850 \\
24.00\end{array}$} \\
\hline 24.90 & 26.40 & 43.30 & 51.60 & 69.60 & 102.00 & 102.00 & 101.90 \\
\hline 55.80 & 55.80 & 55.50 & 56.80 & 60.40 & 71.20 & 80.60 & \\
\hline-1.00 & 53.20 & 52.60 & 59.00 & 62.30 & 63.80 & 76.40 & \\
\hline-1.00 & 56.90 & 57.70 & 58.00 & 60.20 & 69.70 & 76.80 & \\
\hline-1.00 & 54.20 & 57.50 & 58.50 & 66.30 & 60.50 & 86.40 & \\
\hline \multicolumn{8}{|c|}{$\begin{array}{l}617 \quad 196.85 \quad 45 \\
24.00\end{array}$} \\
\hline 24.90 & 26.40 & 43.30 & 51.50 & 69.10 & 101.60 & 101.60 & 101.50 \\
\hline 56.10 & 56.60 & 56.70 & 64.50 & 60.20 & 64.40 & 72.20 & \\
\hline-1.00 & 54.40 & 53.70 & 57.20 & 63.00 & 75.60 & 77.50 & \\
\hline-1.00 & 53.40 & 59.10 & 60.70 & 61.80 & 71.20 & 68.70 & \\
\hline \multirow{2}{*}{\multicolumn{8}{|c|}{$\begin{array}{l}660196.8545 \\
24.00\end{array}$}} \\
\hline & & & & & & & \\
\hline 24.90 & 26.40 & 42.70 & 50.80 & 68.80 & 101.60 & 101.60 & 101.60 \\
\hline 55.70 & 56.10 & 55.90 & 64.30 & 59.20 & 63.90 & 71.70 & \\
\hline-1.00 & 53.80 & 53.10 & 56.60 & 62.30 & 74.90 & 76.60 & \\
\hline-1.00 & 52.90 & 58.40 & 59.70 & 61.20 & 70.80 & 68.50 & \\
\hline-1.00 & 55.80 & 56.10 & 55.70 & 59.80 & 76.20 & 85.40 & \\
\hline \multicolumn{8}{|c|}{$\begin{array}{l}660196.850 \\
24.10\end{array}$} \\
\hline 24.80 & 26.50 & 42.80 & 50.90 & 68.70 & 101.70 & 101.90 & 101.70 \\
\hline 55.30 & 55.40 & 55.10 & 56.20 & 61.90 & 69.30 & 82.30 & \\
\hline-1.00 & 52.60 & 52.10 & 56.90 & 59.80 & 62.20 & 70.30 & \\
\hline
\end{tabular}




\begin{tabular}{|c|c|c|c|c|c|c|c|}
\hline-1.00 & 56.60 & 57.00 & 57.30 & 59.50 & 70.90 & 83.10 & \\
\hline-1.00 & 54.10 & 57.20 & 57.90 & 64.80 & 61.30 & 89.80 & \\
\hline \multicolumn{8}{|c|}{$\begin{array}{l}705196.850 \\
24.30\end{array}$} \\
\hline 25.10 & 26.30 & 42.00 & 50.00 & 67.80 & 101.70 & 101.70 & \multirow[t]{5}{*}{101.70} \\
\hline 53.90 & 53.60 & 53.40 & 54.50 & 59.40 & 66.70 & 79.10 & \\
\hline-1.00 & 51.10 & 51.70 & 55.30 & 58.50 & 61.00 & 68.20 & \\
\hline-1.00 & 54.80 & 55.70 & 56.40 & 58.50 & 67.60 & 81.30 & \\
\hline-1.00 & 52.60 & 56.10 & 56.10 & 64.00 & 59.30 & 88.50 & \\
\hline \multicolumn{8}{|c|}{$\begin{array}{l}705196.85 \quad 45 \\
24.30\end{array}$} \\
\hline 25.10 & 26.50 & 42.30 & 50.20 & 68.10 & 101.70 & 101.90 & \multirow[t]{5}{*}{101.70} \\
\hline 54.80 & 55.00 & 54.10 & 65.80 & 56.40 & 60.50 & 67.80 & \\
\hline-1.00 & 52.70 & 52.20 & 54.80 & 59.60 & 76.30 & 71.40 & \\
\hline-1.00 & 52.70 & 58.20 & 61.00 & 62.30 & 70.90 & 69.70 & \\
\hline-1.00 & 54.80 & 54.50 & 56.10 & 57.60 & 77.20 & 82.90 & \\
\hline \multicolumn{8}{|c|}{$\begin{array}{l}750 \quad 196.85 \quad 45 \\
24.30\end{array}$} \\
\hline 24.80 & 26.40 & 42.00 & 50.20 & 68.00 & 101.70 & 101.70 & \multirow[t]{5}{*}{101.70} \\
\hline 54.60 & 54.50 & 53.60 & 65.80 & 56.00 & 59.80 & 66.90 & \\
\hline-1.00 & 52.50 & 51.60 & 54.50 & 58.80 & 75.30 & 70.60 & \\
\hline-1.00 & 52.80 & 57.90 & 60.40 & 62.50 & 70.40 & 69.20 & \\
\hline-1.00 & 54.40 & 53.90 & 55.90 & 57.00 & 76.60 & 82.20 & \\
\hline \multirow{2}{*}{\multicolumn{8}{|c|}{750196.850}} \\
\hline 24.40 & & & & & & & \\
\hline 25.10 & 26.50 & 41.80 & 50.00 & 67.80 & 102.00 & 102.00 & \multirow[t]{5}{*}{102.00} \\
\hline 54.40 & 52.20 & 57.40 & 54.30 & 73.40 & 57.80 & 87.80 & \\
\hline-1.00 & 52.90 & 53.10 & 53.70 & 54.70 & 60.20 & 69.90 & \\
\hline-1.00 & 49.70 & 55.20 & 54.20 & 64.80 & 69.00 & 79.40 & \\
\hline-1.00 & 55.70 & 54.80 & 62.50 & 60.70 & 59.90 & 87.70 & \\
\hline \multicolumn{8}{|c|}{$795 \quad 196.850$} \\
\hline \multicolumn{8}{|c|}{24.10} \\
\hline 24.60 & 25.80 & 41.00 & 49.00 & 67.00 & 101.10 & 101.10 & \multirow[t]{5}{*}{100.80} \\
\hline 53.10 & 52.40 & 52.80 & 53.30 & 57.90 & 65.70 & 77.60 & \\
\hline-1.00 & 50.10 & 51.40 & 54.30 & 57.40 & 60.00 & 67.00 & \\
\hline-1.00 & 54.50 & 55.00 & 55.90 & 57.20 & 66.30 & 78.80 & \\
\hline-1.00 & 51.90 & 55.40 & 54.40 & 63.60 & 57.80 & 87.80 & \\
\hline \multicolumn{8}{|c|}{$795 \quad 196.85 \quad 45$} \\
\hline 24.60 & 26.00 & 41.00 & 49.00 & 67.00 & 101.10 & 101.30 & \multirow[t]{5}{*}{101.10} \\
\hline 53.20 & 53.50 & 54.50 & 59.10 & 60.00 & 66.90 & 73.20 & \\
\hline-1.00 & 51.40 & 50.50 & 57.30 & 59.40 & 60.50 & 74.30 & \\
\hline-1.00 & 51.20 & 54.80 & 53.30 & 57.70 & 69.90 & 63.90 & \\
\hline-1.00 & 54.10 & 54.40 & 55.00 & 58.90 & 66.80 & 84.40 & \\
\hline \multicolumn{8}{|c|}{$\begin{array}{l}841 \quad 196.85 \quad 45 \\
24.00\end{array}$} \\
\hline 24.50 & 25.90 & 40.90 & 48.80 & 66.90 & 101.00 & 101.00 & \multirow[t]{5}{*}{101.00} \\
\hline 52.90 & 53.10 & 54.20 & 58.80 & 59.80 & 66.10 & 72.80 & \\
\hline-1.00 & 51.40 & 50.20 & 57.30 & 58.50 & 59.80 & 73.00 & \\
\hline-1.00 & 51.00 & 54.40 & 53.30 & 57.20 & 69.10 & 63.40 & \\
\hline-1.00 & 53.70 & 53.40 & 55.00 & 58.60 & 66.50 & 83.60 & \\
\hline \multicolumn{8}{|c|}{$\begin{array}{l}841196.850 \\
24.20\end{array}$} \\
\hline 24.70 & 25.90 & 41.00 & 49.00 & 66.90 & 101.20 & 101.20 & \multirow[t]{2}{*}{101.10} \\
\hline 52.80 & 52.20 & 52.40 & 53.10 & 57.50 & 64.30 & 76.70 & \\
\hline
\end{tabular}


$-1.00$

$-1.00$

$-1.00$
49.90

53.80

51.60
51.40

54.50

55.40
53.70

55.80

53.90
57.20

56.80

63.20
59.80

65.50

57.60

66.20

77.90

87.30 


\section{$26.2 \mathrm{~mm}$ column, $19.05 \mathrm{~mm}$ polystyrene spheres}

\section{Format}

First 4 lines:

\# radial profiles \# radial T/C positions \# wall temperatures \# angular positions

Radial $\mathrm{T} / \mathrm{C}$ positions in $\mathrm{mm}$ from center of bed

Axial T/C positions on tube wall (-ve means calming section) in $\mathrm{mm}$

Tube radius Sphere diameter

Data then follow in blocks:

$\operatorname{Re} \quad \mathrm{L}(\mathrm{mm})$ Angle

$\mathrm{T}_{\text {in }}$ (entrance to calming section)

Tube wall temperature measurements

Radial profiles (-1 means no temperature measured; note temperatures at $r=0.0$ were not used)

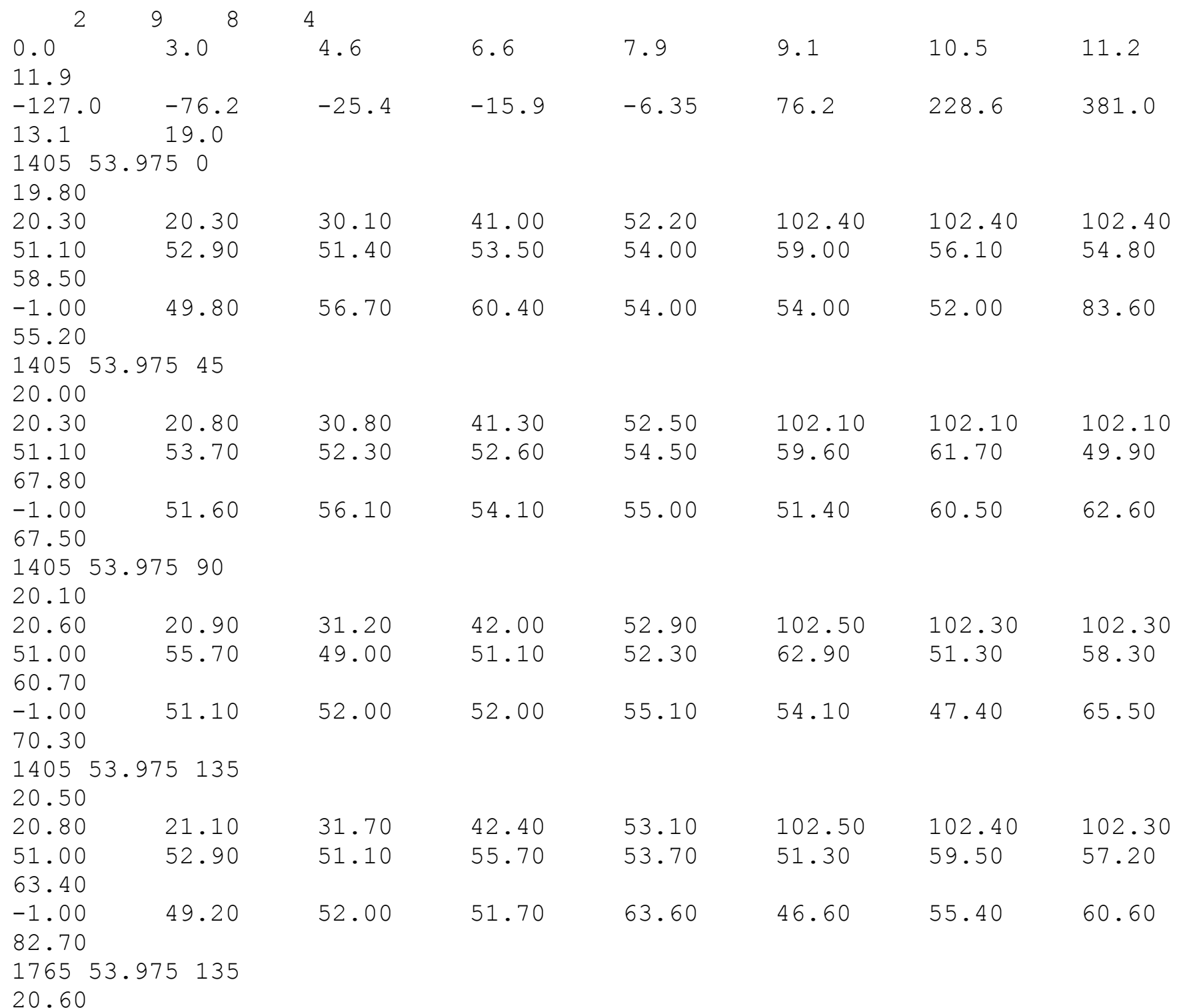




\begin{tabular}{|c|c|c|c|c|c|c|c|}
\hline 21.10 & 21.50 & 31.50 & 41.80 & 51.40 & 102.40 & 102.40 & 102.20 \\
\hline 48.60 & 50.70 & 48.20 & 52.20 & 50.90 & 49.10 & 56.80 & 54.10 \\
\hline $\begin{array}{l}58.80 \\
-1.00\end{array}$ & & & & & & & \\
\hline 76.80 & 46.60 & 49.30 & 49.10 & 60.00 & 44.40 & 52.80 & 57.20 \\
\hline $\begin{array}{l}1765 \\
20.60\end{array}$ & $75 \quad 90$ & & & & & & \\
\hline 21.10 & 21.50 & 31.50 & 41.80 & 51.40 & 102.40 & 102.20 & 102.20 \\
\hline 48.60 & 54.30 & 46.60 & 49.40 & 50.90 & 62.20 & 53.50 & 57.10 \\
\hline 55.50 & & & & & & & \\
\hline-1.00 & 48.90 & 52.80 & 46.60 & 55.70 & 50.20 & 45.30 & 59.50 \\
\hline 57.70 & & & & & & & \\
\hline 17655 & $75 \quad 45$ & & & & & & \\
\hline 20.80 & & & & & & & \\
\hline 21.30 & 21.70 & 31.80 & 42.10 & 51.60 & 102.10 & 102.10 & 102.10 \\
\hline 48.50 & 52.30 & 49.50 & 48.10 & 50.80 & 56.80 & 67.20 & 44.80 \\
\hline 63.50 & & & & & & & \\
\hline-1.00 & 49.60 & 54.90 & 48.60 & 51.30 & 50.10 & 55.30 & 57.60 \\
\hline 52.20 & & & & & & & \\
\hline 17655 & 750 & & & & & & \\
\hline 20.90 & & & & & & & \\
\hline 21.40 & 22.20 & 32.00 & 42.20 & 51.60 & 102.40 & 102.40 & 102.10 \\
\hline 48.30 & 50.50 & 49.90 & 51.00 & 48.50 & 54.20 & 58.10 & 54.90 \\
\hline 56.70 & & & & & & & \\
\hline-1.00 & 47.50 & 55.50 & 62.60 & 55.40 & 55.40 & 50.80 & 83.80 \\
\hline 51.10 & & & & & & & \\
\hline 21345 & 750 & & & & & & \\
\hline 21.10 & & & & & & & \\
\hline 21.90 & 22.40 & 31.70 & 41.50 & 49.90 & 102.10 & 102.10 & 101.80 \\
\hline 46.60 & 47.30 & 47.50 & 47.50 & 52.20 & 55.60 & 50.10 & 48.00 \\
\hline 47.10 & & & & & & & \\
\hline-1.00 & 46.60 & 48.40 & 51.40 & 46.90 & 48.00 & 49.90 & 57.60 \\
\hline 47.60 & & & & & & & \\
\hline 21345 & $75 \quad 45$ & & & & & & \\
\hline 21.40 & & & & & & & \\
\hline 22.10 & 22.90 & 31.90 & 41.80 & 50.40 & 102.60 & 102.30 & 102.10 \\
\hline 47.40 & 47.80 & 46.90 & 46.90 & 47.50 & 51.70 & 54.00 & 47.50 \\
\hline 51.70 & & & & & & & \\
\hline-1.00 & 48.10 & 50.50 & 54.20 & 51.70 & 49.00 & 48.30 & 55.80 \\
\hline 63.20 & & & & & & & \\
\hline 21345 & $75 \quad 90$ & & & & & & \\
\hline 21.30 & & & & & & & \\
\hline 22.10 & 22.70 & 31.90 & 41.70 & 50.10 & 102.40 & 102.30 & 102.30 \\
\hline 47.30 & 48.40 & 46.60 & 51.20 & 50.20 & 52.30 & 61.70 & 52.00 \\
\hline 51.90 & & & & & & & \\
\hline-1.00 & 47.50 & 50.50 & 46.30 & 49.10 & 47.70 & 51.60 & 50.70 \\
\hline 53.10 & & & & & & & \\
\hline 21345 & $75 \quad 135$ & & & & & & \\
\hline 21.30 & & & & & & & \\
\hline 22.00 & 22.70 & 31.80 & 41.60 & 50.20 & 102.40 & 102.20 & 102.20 \\
\hline 46.90 & 49.90 & 49.20 & 48.70 & 47.60 & 60.30 & 47.90 & 51.60 \\
\hline 48.30 & & & & & & & \\
\hline-1.00 & 46.90 & 47.50 & 49.80 & 51.10 & 52.50 & 50.10 & 53.10 \\
\hline 54.10 & & & & & & & \\
\hline 1125 & 135 & & & & & & \\
\hline
\end{tabular}


$23.00 \quad 31.50$

48.20

46.90

$-1.00$

52.00

$2512 \quad 53.975 \quad 90$

21.50

22.20

45.60

53.00

$-1.00$

51.90

$2512 \quad 53.975 \quad 45$

21.60

22.20

45.90

48.50

$-1.00$

58.10

251253.9750

21.60

22.30

45.60

50.70

$-1.00$

53.20

283953.9750

24.40

24.80

45.90

58.90

$-1.00$

52.20

$2839 \quad 53.975 \quad 45$

24.10

24.80

46.00

48.40

$-1.00$

53.90

$283953.975 \quad 90$

24.30

24.80

46.50

50.40

$-1.00$

51.10

$2839 \quad 53.975 \quad 135$

24.40

25.10

45.90

47.30

$-1.00$

49.60

45.80

23.00

46.40

46.30

23.00

46.30

46.30

23.00

46.30

45.70

25.10

45.70

47.10

25.30

45.90

46.40

31.60

46.30

45.80

31.60

45.30

48.20

33.00

46.60

45.70

33.10

45.90

48.30

25.30

48.30

33.20

45.20

48.20

46.90

25.60

48.70

45.50

33.30

45.90

45.90
$41.20 \quad 48.80$

46.90

48.30

54.10

40.90

44.80

48.70

45.40

51.30

41.20

46.30

48.20

48.90

53.10

47.10

42.30

46.10

49.80

54.90

47.50

45.90

42.30

45.00

49.80

45.30

54.80

50.70

42.40

49.40

50.10

49.80

45.30

47.70

49.20

42.40

46.90

49.60

46.90

101.40

59.10

101.40

47.20

101.10

49.20

48.50

50.60

47.80

53.30 
$3163 \quad 53.975 \quad 135$

24.30

25.20

44.80

46.20

$-1.00$

48.30

$3163 \quad 53.97590$

24.40

25.20

45.50

50.60

$-1.00$

49.70

25.70

47.50

33.20

44.70

41.70

45.90

48.50

101.20

100.90

100.90

46.20

57.80

46.20

48.50

$3163 \quad 53.975 \quad 45$

24.60

25.40

45.20

25.90

44.50

47.80

47.60

49.50

46.70

52.70

45.90

$-1.00$

45.50

33.10

45.80

41.90

42.00

49.00

48.50

48.80

101.20

101.20

100.90

44.70

50.40

58.80

48.70

44.70

46.70

46.40

48.70

48.90

48.30

45.00

46.20

45.00

48.70

46.40

101.40

50.10

101.10

47.30

101.10

51.70

47.90

51.00

46.60

48.70

$3163 \quad 53.9750$

24.80

25.50

26.00

33.40

42.50

49.50

54.10

101.20

52.00

101.20

48.50

101.20

45.80
57.30

46.10

45.80

45.50

46.10

50.60

46.70

$-1.00$

47.10

45.70

47.70

45.50

$3491 \quad 53.975 \quad 0$

24.20

25.30

25.80

32.80

41.40

47.30

43.80

51.90

101.10

51.30

101.00

46.10

100.90

55.40

$-1.00$

44.10

43.80

46.00

43.40

44.20

48.40

45.00

48.30

$3491 \quad 53.975 \quad 45$

24.60

25.20

43.60

45.60

$-1.00$

50.90

$3491 \quad 53.975 \quad 90$

25.10

25.50

44.40

50.20

$-1.00$

25.70

32.70

41.20

47.10

43.10

43.30

100.80

47.20

100.80

100.80

43.90

52.20

48.20

48.70

47.50

47.50

46.60

4.20

44.90

59.70

48.60

$3491 \quad 53.975 \quad 135$

25.20

25.60

44.80

25.90

32.80

32.50

41.40

48.10

102.50

102.50

102.50

47.50

48.20

49.60

57.80

48.40

46.60

48.20

44.70

43.80

45.90

45.80

48.10

47.20

41.60

48.50

102.80

102.60

102.60

46.30

57.40

45.90

48.20 


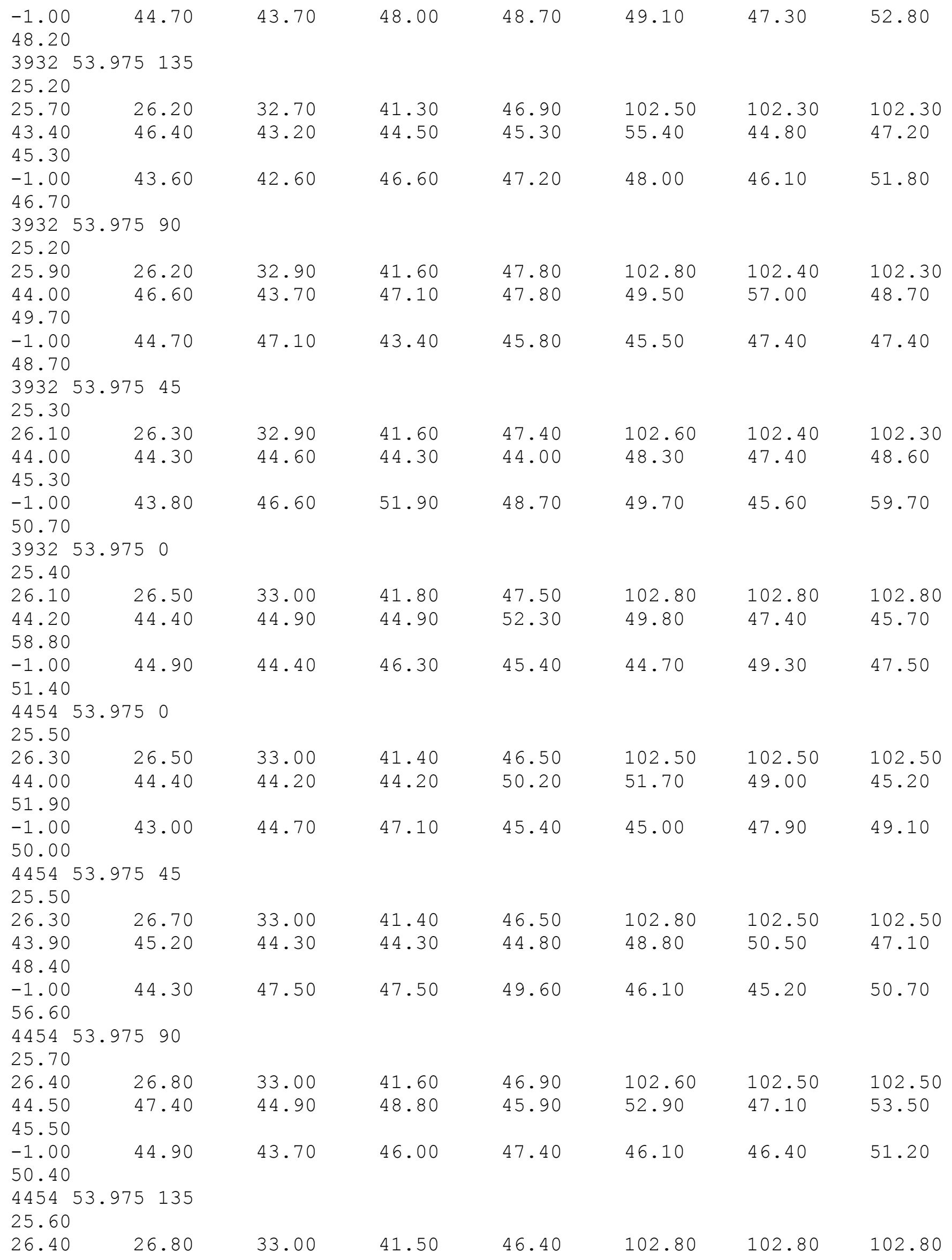




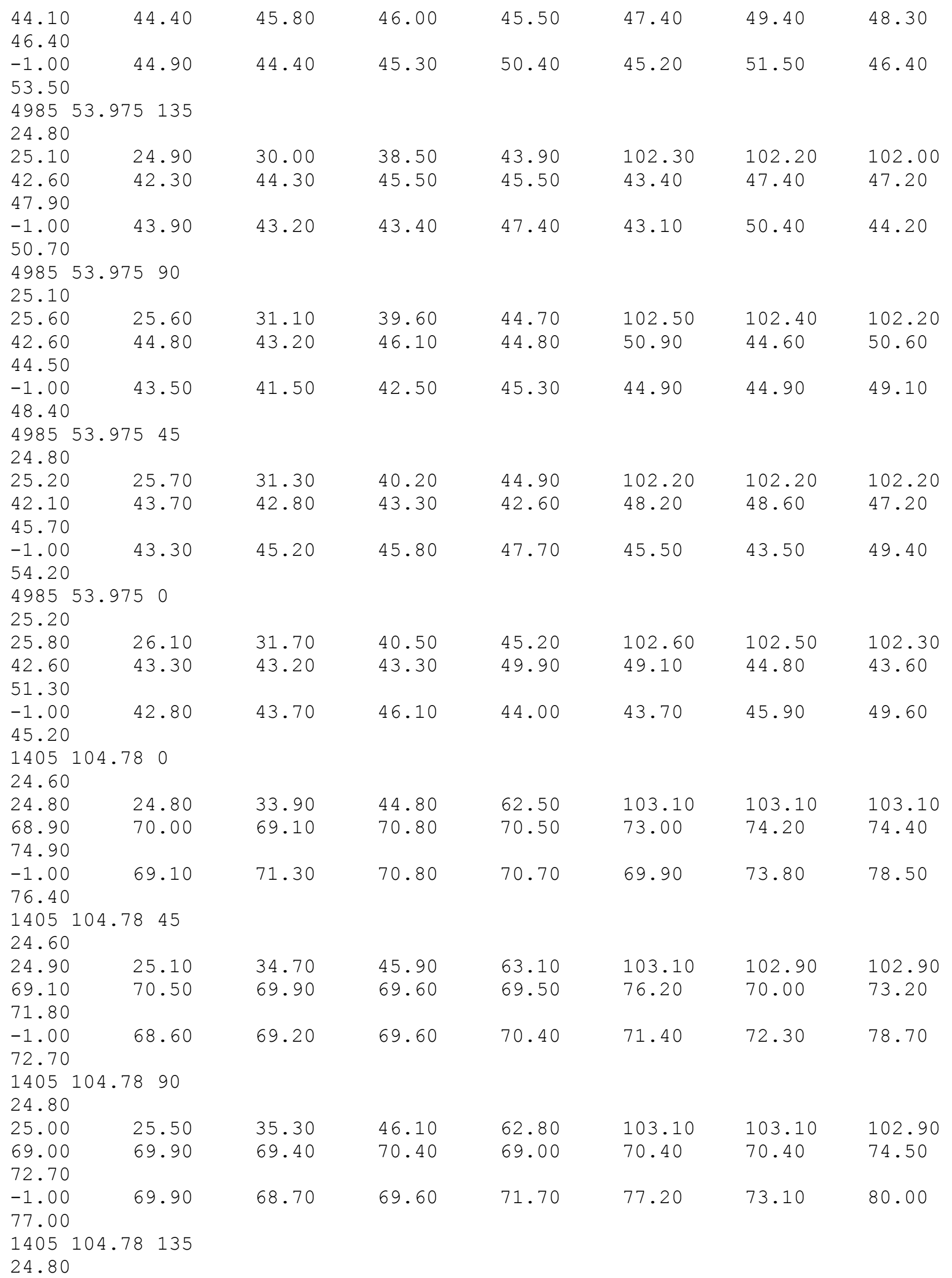




\begin{tabular}{|c|c|c|c|c|c|c|c|}
\hline 25.20 & 25.70 & 35.60 & 46.70 & 63.70 & 103.10 & 103.10 & 103.10 \\
\hline 69.20 & 69.70 & 70.00 & 72.00 & 72.70 & 71.90 & 70.10 & 77.90 \\
\hline 75.00 & & & & & & & \\
\hline-1.00 & 70.70 & 69.30 & 69.40 & 71.00 & 72.80 & 74.20 & 79.30 \\
\hline 70.10 & & & & & & & \\
\hline 1765 & $78 \quad 135$ & & & & & & \\
\hline 24.80 & & & & & & & \\
\hline 25.20 & 25.70 & 35.10 & 45.80 & 61.30 & 103.10 & 103.00 & 102.80 \\
\hline 66.00 & 66.90 & 66.60 & 68.80 & 69.30 & 68.60 & 67.20 & 74.60 \\
\hline 71.70 & & & & & & & \\
\hline-1.00 & 67.30 & 66.10 & 66.60 & 67.90 & 69.30 & 70.00 & 76.30 \\
\hline 67.40 & & & & & & & \\
\hline 1765 & 7890 & & & & & & \\
\hline 24.80 & & & & & & & \\
\hline 25.60 & 26.00 & 35.40 & 46.10 & 61.90 & 103.10 & 103.10 & 102.90 \\
\hline 66.50 & 68.00 & 67.10 & 67.50 & 67.40 & 67.80 & 69.30 & 70.60 \\
\hline 72.50 & & & & & & & \\
\hline-1.00 & 67.10 & 66.60 & 66.60 & 69.30 & 71.10 & 71.10 & 74.60 \\
\hline 73.70 & & & & & & & \\
\hline 1765 & $78 \quad 45$ & & & & & & \\
\hline 24.90 & & & & & & & \\
\hline 25.70 & 26.20 & 35.50 & 45.90 & 61.60 & 103.10 & 103.10 & 102.90 \\
\hline 66.60 & 67.50 & 67.50 & 68.40 & 67.00 & 71.70 & 69.40 & 74.00 \\
\hline 68.90 & & & & & & & \\
\hline-1.00 & 66.40 & 68.00 & 67.30 & 67.90 & 70.20 & 67.70 & 77.60 \\
\hline 71.50 & & & & & & & \\
\hline 1765 & 780 & & & & & & \\
\hline 25.20 & & & & & & & \\
\hline 25.70 & 26.20 & 35.50 & 45.90 & 61.40 & 102.80 & 102.80 & 102.80 \\
\hline 66.00 & 67.20 & 66.30 & 68.20 & 67.80 & 70.20 & 71.40 & 70.30 \\
\hline 71.60 & & & & & & & \\
\hline-1.00 & 66.30 & 68.50 & 68.40 & 67.80 & 67.20 & 70.10 & 75.20 \\
\hline 73.30 & & & & & & & \\
\hline 2134 & 80 & & & & & & \\
\hline 25.30 & & & & & & & \\
\hline 25.80 & 26.50 & 35.30 & 45.90 & 60.30 & 103.20 & 103.20 & 103.20 \\
\hline 64.40 & 65.00 & 64.00 & 66.00 & 65.80 & 68.50 & 69.90 & 68.70 \\
\hline 69.70 & & & & & & & \\
\hline-1.00 & 64.00 & 66.30 & 66.30 & 66.00 & 65.50 & 68.40 & 73.20 \\
\hline 71.60 & & & & & & & \\
\hline 2134 & $78 \quad 45$ & & & & & & \\
\hline 25.10 & & & & & & & \\
\hline 25.80 & 26.50 & 35.30 & 45.60 & 60.20 & 103.40 & 103.40 & 103.10 \\
\hline 64.40 & 65.80 & 65.80 & 66.30 & 64.70 & 69.70 & 67.00 & 71.80 \\
\hline 66.60 & & & & & & & \\
\hline-1.00 & 64.40 & 66.10 & 65.30 & 65.70 & 67.90 & 66.20 & 74.70 \\
\hline 68.70 & & & & & & & \\
\hline 2134 & 7890 & & & & & & \\
\hline 25.30 & & & & & & & \\
\hline 25.80 & 26.70 & 35.10 & 45.40 & 59.90 & 103.20 & 103.20 & 103.20 \\
\hline 64.30 & 66.00 & 65.10 & 65.60 & 65.20 & 66.30 & 66.90 & 68.70 \\
\hline 68.80 & & & & & & & \\
\hline-1.00 & 65.30 & 64.60 & 64.90 & 67.30 & 71.00 & 68.40 & 73.90 \\
\hline 72.50 & & & & & & & \\
\hline 2134 & 135 & & & & & & \\
\hline
\end{tabular}


25.30

26.00

64.30

69.50

$-1.00$

66.60

$2512 \quad 104.78 \quad 135$

25.40

26.00

62.70

67.70

$-1.00$

$26.70 \quad 35.30$

64.60

64.60

45.40

59.70

102.90

102.90

102.80

66.90

67.20

66.80

65.40

74.30

65.00

$2512 \quad 104.78 \quad 90$

25.50

26.20

26.70

63.70

64.10

66.30

67.50

68.10

72.20

62.80

64.60

35.00

34.90

45.20

58.50

65.90

65.40

102.50

102.50

102.30

63.30

65.00

63.60

72.30

67.90

$-1.00$

63.20

62.60

63.20

64.50

65.90

66.20

70.50

70.10

$\begin{array}{lll}2512 & 104.78 \quad 45\end{array}$

25.60

26.20

26.80

34.90

44.90

45.10

58.50

63.70

103.10

103.20

103.10

63.70

65.10

65.60

66.40

63.10

62.90

65.50

67.30

67.40

70.90

62.60

63.70

64.40

58.20

63.00

102.60

102.60

102.40

64.60

$-1.00$

62.50

63.20

63.50

67.60

66.00

70.20

66.40

64.10

65.80

64.40

72.20

$2512 \quad 104.78 \quad 0$

25.80

26.40

27.00

35.10

45.20

58.50

64.00

64.00

102.80

102.60

102.40

62.70

63.40

62.50

66.30

67.90

66.90

$-1.00$

62.50

64.40

64.60

64.30

63.80

66.40

71.40

$2839104.78 \quad 0$

25.80

26.60

61.60

27.00

35.00

44.80

57.20

63.50

62.90

103.20

103.20

103.20

66.60

$-1.00$

61.60

63.80

64.00

63.20

65.00

67.20

66.00

68.80

$2839 \quad 104.78 \quad 45$

25.90

26.70

27.10

34.80

44.80

57.30

62.80

65.50

70.50

61.60

63.00

63.00

63.00

62.30

103.30

103.30

102.90

63.90

61.60

62.70

62.40

62.50

67.40

64.00

67.40

64.60

$2839104.78 \quad 90$

25.90

26.50

61.70

27.10

35.10

44.80

57.50

64.60

65.40

70.30

67.60

$-1.00$

63.50

62.60

62.60

63.00

103.30

103.30

103.30

62.20

62.00

62.20

64.30

64.30

64.30

64.80

69.00 
$2839 \quad 104.78 \quad 135$

26.00

26.60

62.00

27.20

62.20

35.10

45.00

57.60

103.40

103.40

103.40

72.60

$-1.00$

62.60

62.20

64.40

66.10

64.00

64.40

70.90

64.40

$3163 \quad 104.78 \quad 135$

26.00

26.80

27.20

61.90

62.20

63.10

64.40

64.90

70.00

60.70

61.10

34.70

61.10

44.50

63.50

56.10

64.70

103.70

103.50

103.40

71.20

$-1.00$

61.60

61.10

61.10

62.00

62.60

63.40

69.70

63.40

$3163 \quad 104.78 \quad 90$

26.00

26.80

27.20

34.70

44.50

56.70

63.40

63.50

68.80

60.80

62.30

61.40

61.40

62.00

103.50

103.40

103.40

66.70

$-1.00$

60.90

60.70

60.90

63.40

63.30

63.50

64.30

68.00

$\begin{array}{lll}3163 & 104.78 \quad 45\end{array}$

26.00

26.80

27.30

34.80

44.30

56.10

61.10

103.50

103.50

103.30

60.70

61.70

62.10

66.20

63.90

67.90

62.50

60.30

61.70

61.00

61.60

63.90

63.00

69.00

64.40

$\begin{array}{lll}3163 & 104.78 \quad 0\end{array}$

26.00

26.80

27.30

34.80

44.30

56.10

62.00

103.50

103.50

103.30

60.70

60.70

62.30

64.10

66.30

65.00

65.50

60.40

62.70

62.90

62.10

61.70

64.40

69.20

67.80

$3491 \quad 104.78 \quad 0$

25.60

26.50

27.00

34.00

43.80

54.00

60.40

59.80

103.50

103.30

103.00

58.60

59.40

58.60

62.10

64.40

63.30

$-1.00$

58.40

60.70

60.70

60.20

59.80

62.30

67.10

$3491 \quad 104.78 \quad 45$

25.90

26.50

59.00

27.00

34.30

43.80

54.40

60.90

59.80

103.40

103.30

103.20

61.10

$-1.00$

59.10

60.10

59.60

60.10

65.00

61.80

65.00

62.20

$3491 \quad 104.78 \quad 90$

25.90

26.50

27.00

34.10

43.60

54.30

62.20

62.80

66.70

58.80

60.30

59.40

59.80

60.10

103.30

103.10

103.00

64.80 


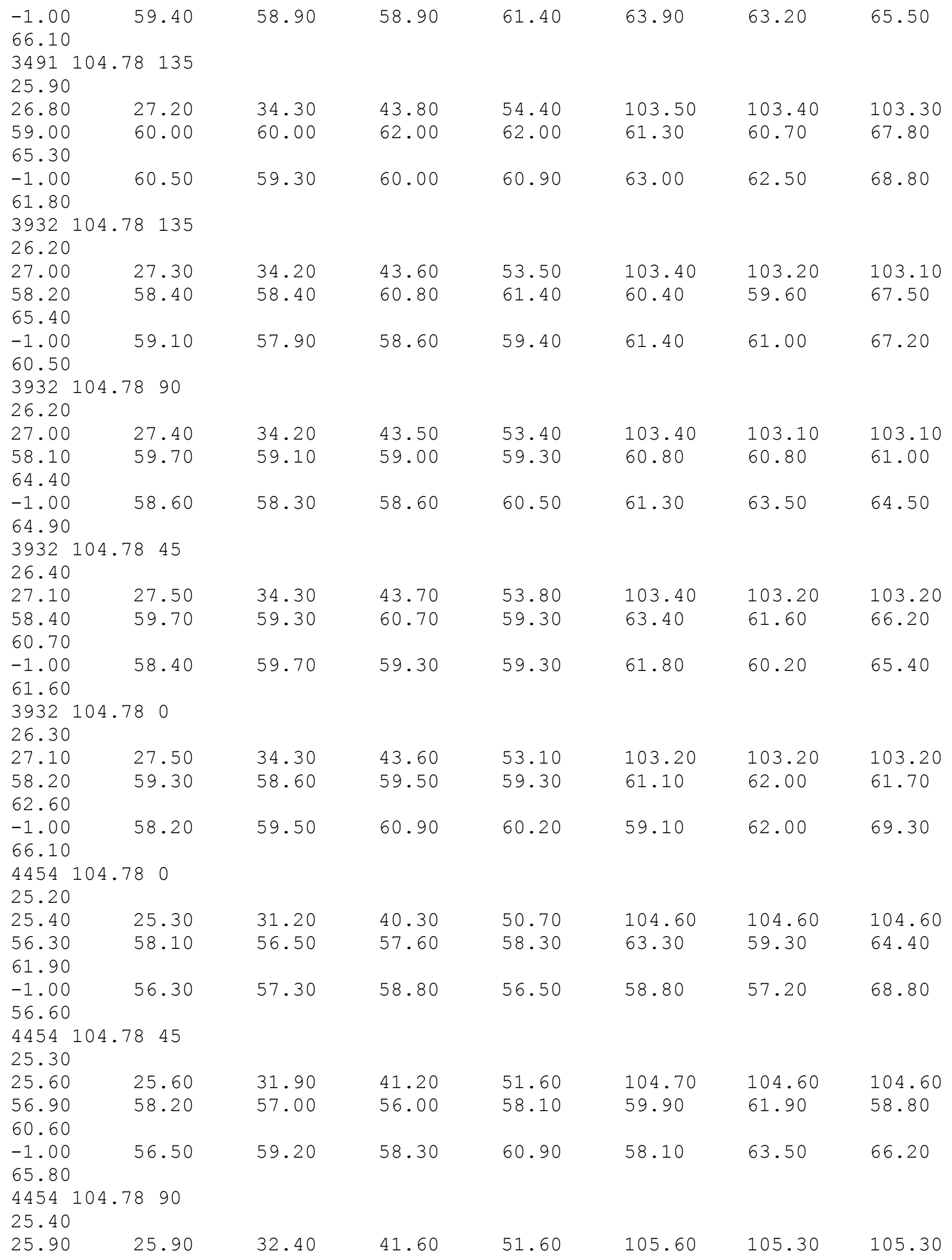




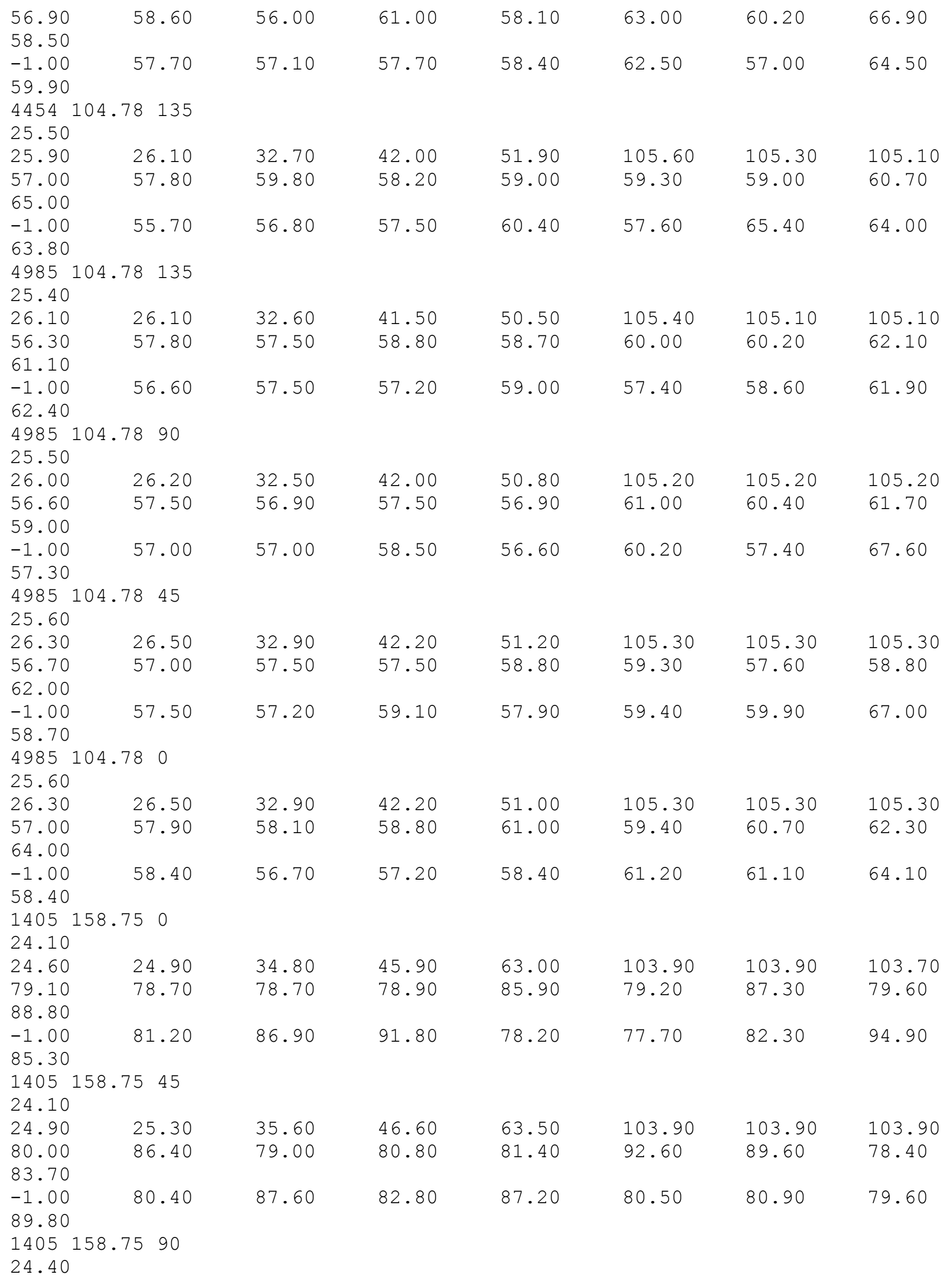




\begin{tabular}{|c|c|c|c|c|c|c|c|}
\hline 25.10 & 25.70 & 36.10 & 47.00 & 63.80 & 103.90 & 103.90 & 103.70 \\
\hline 78.70 & 84.90 & 77.80 & 82.30 & 80.50 & 91.20 & 89.60 & 79.60 \\
\hline $\begin{array}{l}80.50 \\
-1.00\end{array}$ & & & & & & & \\
\hline $\begin{array}{l}-1.00 \\
90.60\end{array}$ & 79.10 & 87.00 & 81.90 & 87.20 & 80.90 & 79.60 & 80.90 \\
\hline $\begin{array}{l}1405 \\
24.30\end{array}$ & $75 \quad 135$ & & & & & & \\
\hline 25.10 & 25.70 & 36.10 & 46.80 & 63.80 & 103.90 & 103.70 & 103.70 \\
\hline 78.50 & 84.00 & 78.20 & 84.90 & 80.30 & 87.80 & 85.90 & 82.80 \\
\hline 80.50 & & & & & & & \\
\hline-1.00 & 79.10 & 83.70 & 82.30 & 86.20 & 82.80 & 79.10 & 84.60 \\
\hline 91.60 & & & & & & & \\
\hline 1765 & $75 \quad 135$ & & & & & & \\
\hline $\begin{array}{l}24.40 \\
25.30\end{array}$ & 25.80 & 35.80 & 46.30 & 62.00 & 104.00 & 103.90 & 103.70 \\
\hline 75.90 & 81.40 & 75.50 & 82.30 & 77.70 & 85.00 & 84.00 & 80.20 \\
\hline 77.70 & & & & & & & \\
\hline-1.00 & 76.40 & 81.40 & 79.40 & 84.10 & 80.00 & 76.40 & 82.20 \\
\hline 88.70 & & & & & & & \\
\hline 1765 & $75 \quad 90$ & & & & & & \\
\hline 24.40 & & & & & & & \\
\hline 25.40 & 25.90 & 35.90 & 46.30 & 62.00 & 103.90 & 103.90 & 103.60 \\
\hline 76.10 & 82.80 & 75.60 & 81.20 & 78.20 & 88.30 & 86.80 & 77.30 \\
\hline 77.80 & & & & & & & \\
\hline-1.00 & 76.50 & 84.30 & 79.50 & 84.60 & 77.80 & 77.30 & 78.20 \\
\hline 88.30 & & & & & & & \\
\hline 1765 & $75 \quad 45$ & & & & & & \\
\hline 24.60 & & & & & & & \\
\hline 25.40 & 26.00 & 35.40 & 45.60 & 60.80 & 103.70 & 103.60 & 103.40 \\
\hline 76.30 & 81.40 & 75.90 & 76.90 & 78.10 & 87.20 & 84.50 & 75.00 \\
\hline 80.80 & & & & & & & \\
\hline-1.00 & 77.20 & 83.70 & 79.80 & 82.30 & 76.80 & 77.20 & 75.80 \\
\hline 86.80 & & & & & & & \\
\hline 1765 & 750 & & & & & & \\
\hline 24.30 & & & & & & & \\
\hline 25.20 & 25.80 & 35.40 & 45.70 & 61.60 & 103.50 & 103.50 & 103.50 \\
\hline 76.00 & 77.50 & 76.40 & 77.00 & 84.70 & 77.80 & 88.20 & 77.10 \\
\hline 86.10 & & & & & & & \\
\hline-1.00 & 80.60 & 87.60 & 87.10 & 79.40 & 76.10 & 78.80 & 86.70 \\
\hline 82.80 & & & & & & & \\
\hline 2134 & 750 & & & & & & \\
\hline 24.60 & & & & & & & \\
\hline 25.50 & 26.20 & 35.00 & 44.80 & 59.40 & 103.50 & 103.50 & 103.40 \\
\hline 72.70 & 74.60 & 73.30 & 74.10 & 81.40 & 75.30 & 84.60 & 74.50 \\
\hline 83.40 & & & & & & & \\
\hline-1.00 & 77.00 & 84.30 & 83.30 & 76.60 & 73.20 & 75.90 & 82.90 \\
\hline 80.00 & & & & & & & \\
\hline 2134 & $75 \quad 45$ & & & & & & \\
\hline 24.60 & & & & & & & \\
\hline 25.50 & 26.20 & 35.00 & 45.20 & 60.10 & 103.50 & 103.50 & 103.30 \\
\hline 75.20 & 78.10 & 74.90 & 75.80 & 80.60 & 80.80 & 83.40 & 76.70 \\
\hline 78.40 & & & & & & & \\
\hline-1.00 & 74.90 & 83.40 & 83.50 & 74.70 & 75.60 & 75.60 & 83.40 \\
\hline 75.80 & & & & & & & \\
\hline
\end{tabular}


24.70

25.60

73.70

26.30

35.10

45.30

60.00

104.20

104.20

104.20

75.60

79.70

73.50

79.90

75.70

84.70

80.60

76.50

$-1.00$

73.90

81.60

75.30

81.50

76.00

73.70

77.40

83.40

$2134 \quad 158.75 \quad 135$

24.90

25.10

73.90

24.90

32.70

77.00

75.80

42.80

77.50

58.40

74.40

102.80

102.60

102.60

74.40

$-1.00$

73.80

75.00

74.80

78.50

80.20

73.80

79.90

80.60

$2512 \quad 158.75 \quad 135$

24.90

25.20

25.40

33.40

43.20

57.50

72.60

102.60

78.60

102.60

72.20

102.60

72.20

72.30

73.40

73.20

76.80

75.80

72.60

78.40

$-1.00$

78.90

$2512 \quad 158.75 \quad 90$

25.40

25.70

72.40

26.00

33.80

43.90

57.90

74.30

102.80

102.80

102.80

76.40

79.10

76.50

76.50

74.20

72.90

77.30

72.30

77.60

72.80

72.80

74.20

77.90

$\begin{array}{lll}2512 & 158.75 \quad 45\end{array}$

25.70

25.70

72.40

26.10

34.10

44.40

58.20

77.00

102.80

81.10

102.80

81.30

102.80

73.50

$-1.00$

72.50

79.10

77.70

72.70

75.90

72.80

76.00

73.00

2512158.750

25.70

25.70

72.40

73.50

$-1.00$

73.00

$2839158.75 \quad 0$

25.40

25.90

70.80

71.90

$-1.00$

26.10

34.10

44.40

58.20

77.00

102.80

81.10

102.80

81.30

102.80

72.90

72.80

76.00

72.80

76.10

78.90

77.60

(76.00

10

34.30

44.10

72.00

57.30

102.60

77.80

102.60

102.60

76.40

78.70

71.90

77.00

72.90

71.40

73.30

79.20

74.90

$2839 \quad 158.75 \quad 45$

25.70

26.20

71.30

72.30

$-1.00$

72.00

26.70

34.60

44.40

57.30

102.80

102.80

102.80

71.60

74.90

78.80

81.60

75.20

76.60

72.00

74.30

71.60

77.90 
$2839158.75 \quad 90$

25.60

26.10

70.80

72.60

26.50

76.40

34.30

44.10

57.00

102.60

102.60

102.60

$76.10 \quad 72.80$

77.20

73.10

76.70

$-1.00$

71.40

76.90

70.50

76.70

72.20

71.30

73.50

76.70

$2839 \quad 158.75 \quad 135$

26.10

26.40

27.00

34.60

75.20

71.60

44.30

77.00

57.40

102.30

102.30

102.30

70.40

70.60

73.50

72.50

71.50

77.50

70.80

81.70

$-1.00$

76.60

$3163 \quad 158.75 \quad 135$

24.80

25.00

24.80

68.50

71.30

31.60

40.90

76.60

73.90

70.90

75.20

69.00

$-1.00$

68.40

70.40

73.10

54.30

100.90

100.60

100.60

69.00

74.30

68.50

75.90

74.50

69.00

69.30

73.10

72.20

69.00

72.60

$\begin{array}{lll}3163 & 158.75 \quad 90\end{array}$

25.10

25.30

25.40

32.30

41.50

72.70

54.30

70.30

101.10

75.00

100.80

100.90

68.10

68.10

69.70

74.40

68.70

76.70

70.00

$-1.00$

68.20

73.80

74.60

$3163 \quad 158.75 \quad 45$

25.60

25.70

68.10

69.50

$-1.00$

26.00

33.00

42.10

54.40

73.20

100.80

76.00

100.80

100.80

68.20

69.70

68.80

70.90

69.30

70.50

69.50

74.40

74.00

3163158.750

25.40

25.60

68.10

69.70

$-1.00$

71.00

$3491 \quad 158.75 \quad 0$

25.10

25.70

66.60

68.10

$-1.00$

25.90

32.90

42.30

68.70

54.40

74.00

76.80

74.90

100.70

100.70

68.50

73.90

69.40

69.30

75.20

68.80

73.40

6.40

70.50

75.50

69.30

$3491 \quad 158.75 \quad 45$

25.20

25.70

26.00

32.70

32.70

41.90

53.20

72.00

100.80

100.80

100.80

67.10

72.90

73.40

67.20

66.30

70.60

66.40

71.70

67.90

67.90

69.10

73.40

67.70

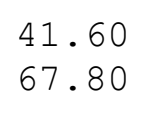

52.60

100.80

100.60

100.60

71.80

75.50

70.00 


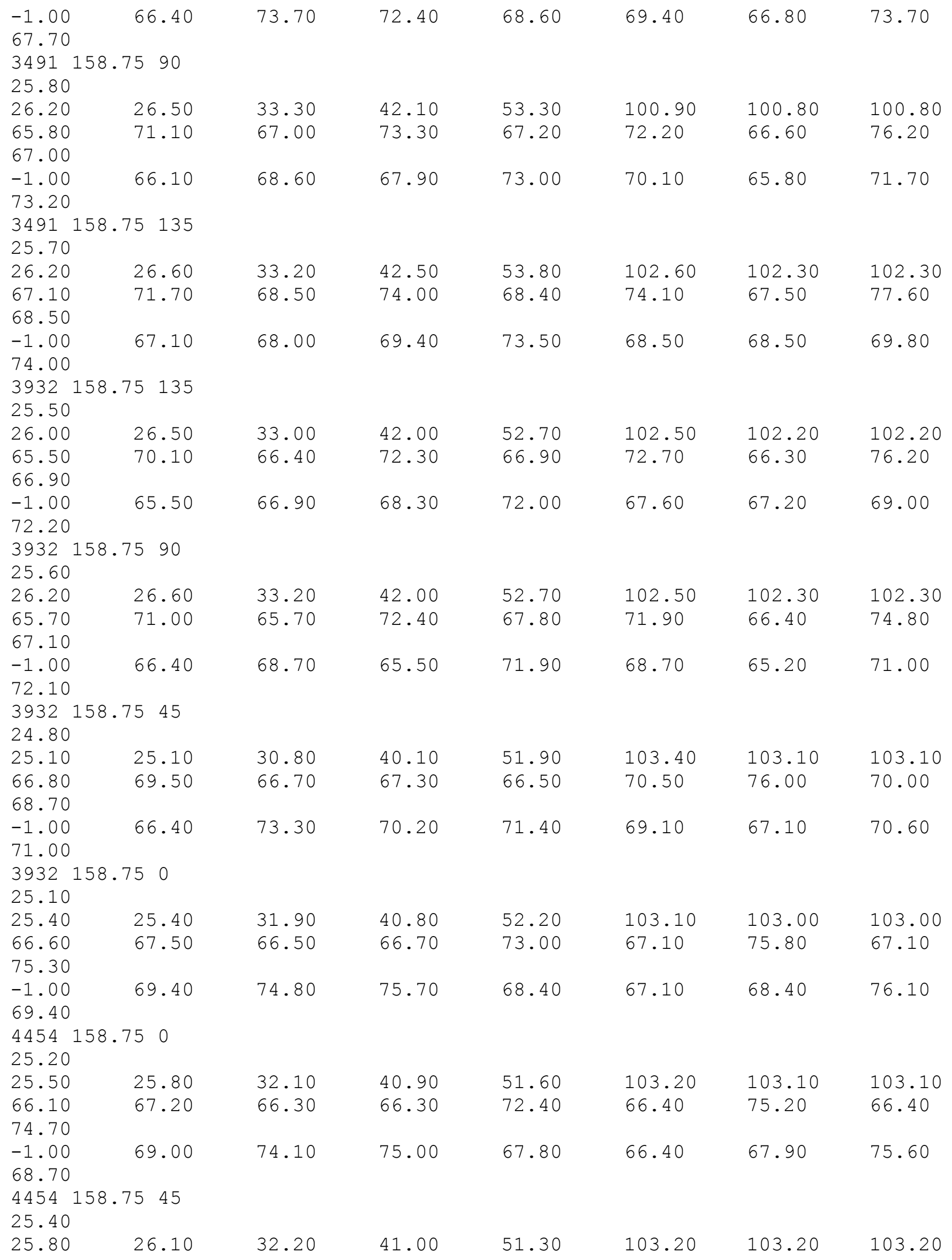




\begin{tabular}{|c|c|c|c|c|c|c|c|}
\hline $\begin{array}{l}65.70 \\
71.40\end{array}$ & 68.60 & 65.20 & 66.60 & 72.80 & 70.50 & 73.70 & 66.90 \\
\hline $\begin{array}{l}-1.00 \\
68.20\end{array}$ & 68.70 & 72.60 & 74.60 & 66.00 & 66.40 & 67.20 & 76.50 \\
\hline $\begin{array}{l}4454 \\
25.40\end{array}$ & 7590 & & & & & & \\
\hline 25.80 & 26.10 & 32.40 & 41.40 & 51.90 & 103.20 & 103.20 & 103.20 \\
\hline $\begin{array}{l}66.60 \\
70.20\end{array}$ & 70.40 & 65.80 & 69.10 & 67.50 & 74.30 & 74.00 & 67.90 \\
\hline $\begin{array}{l}-1.00 \\
73.10 \\
4454 \\
25.40\end{array}$ & $\begin{array}{l}65.40 \\
75 \quad 135\end{array}$ & 71.80 & 66.70 & 72.50 & 66.60 & 68.80 & 67.90 \\
\hline 25.80 & 26.10 & 32.40 & 41.00 & 51.00 & 103.40 & 103.20 & 103.20 \\
\hline $\begin{array}{l}65.50 \\
66.00\end{array}$ & 69.00 & 65.40 & 69.90 & 66.00 & 73.20 & 69.60 & 68.90 \\
\hline $\begin{array}{l}-1.00 \\
71.00 \\
4985 \\
25.50\end{array}$ & $\begin{array}{l}65.80 \\
75 \quad 135\end{array}$ & 68.50 & 65.80 & 70.50 & 68.70 & 66.40 & 69.50 \\
\hline 26.10 & 26.30 & 32.40 & 41.00 & 50.50 & 103.20 & 103.20 & 103.20 \\
\hline $\begin{array}{l}64.70 \\
65.30\end{array}$ & 68.60 & 64.60 & 69.10 & 65.30 & 73.00 & 68.80 & 67.90 \\
\hline $\begin{array}{l}-1.00 \\
70.30 \\
4985 \\
25.50\end{array}$ & $\begin{array}{l}65.00 \\
5 \quad 90\end{array}$ & 67.80 & 65.00 & 70.20 & 67.90 & 65.90 & 68.80 \\
\hline 26.20 & 26.40 & 32.50 & 41.10 & 50.60 & 103.20 & 103.10 & 103.10 \\
\hline $\begin{array}{l}65.10 \\
68.90\end{array}$ & 69.00 & 65.50 & 68.70 & 66.60 & 71.70 & 71.70 & 67.50 \\
\hline $\begin{array}{l}-1.00 \\
71.20 \\
4985 \\
25.70\end{array}$ & $\begin{array}{l}64.80 \\
5 \quad 45\end{array}$ & 70.10 & 65.00 & 70.80 & 65.50 & 67.60 & 67.10 \\
\hline 26.20 & 26.70 & 32.70 & 41.10 & 50.40 & 103.30 & 103.10 & 103.10 \\
\hline $\begin{array}{l}64.60 \\
66.40\end{array}$ & 68.40 & 64.50 & 65.00 & 64.60 & 69.40 & 70.20 & 68.30 \\
\hline $\begin{array}{l}-1.00 \\
69.20 \\
4985 \\
25.80\end{array}$ & $\begin{array}{l}64.20 \\
750\end{array}$ & 69.90 & 70.20 & 69.40 & 67.40 & 65.10 & 67.90 \\
\hline 26.30 & 26.80 & 32.80 & 41.20 & 50.50 & 103.40 & 103.20 & 103.20 \\
\hline $\begin{array}{l}64.40 \\
66.10\end{array}$ & 68.40 & 65.00 & 65.00 & 65.20 & 69.60 & 70.20 & 66.60 \\
\hline $\begin{array}{l}-1.00 \\
66.00\end{array}$ & 64.10 & 69.80 & 70.10 & 67.50 & 67.70 & 66.70 & 72.70 \\
\hline
\end{tabular}

